\title{
Permian-Triassic palynostratigraphy in Mailaram area, Godavari Graben, Andhra Pradesh, India
}

\author{
NeERJA Jha and Neha Aggarwal* \\ Birbal Sahni Institute of Palaeobotany, 53 University Road, Lucknow 226 007, India. \\ *Corresponding author. e-mail: neha_264840@yahoo.co.in
}

Palynological investigations of five borecores, viz., GAM-3, GAM-6, GAM-7, GAM-8 and GAM-10 from Mailaram area have suggested the occurrence of complete Lower Gondwana succession in Mailaram area. Total nine palynozones have been identified on the basis of dominance, sub-dominance and appearance of various palynotaxa. These palynozones belong to Talchir, Upper Karharbari and Barakar (Early Permian), Raniganj (Late Permian) and Panchet (Early Triassic) palynofloras of Indian Gondwana. The oldest Palynozone-1 demonstrated in borecore GAM-6 (331.4-500 m) and borecore GAM-10 (505.66$581.55 \mathrm{~m}$ ), is characterized by the dominance of Parasaccites and sub-dominance of Plicatipollenites belongs to Talchir Palynoflora; Palynozone-2 identified in borecore GAM-7 (667-749 m) and borecore GAM-8 $(89.75 \mathrm{~m})$ is characterized by the dominance of Parasaccites and sub-dominance of Scheuringipollenites which belongs to Upper Karharbari Palynoflora. Palynozone-3, identified in borecore GAM-6 $(149.7-240.05 \mathrm{~m})$ and borecore GAM-8 $(84.95 \mathrm{~m})$, is characterized by the dominance of Scheuringipollenites akin to Scheuringipollenites zone of Barakar palynoflora; Palynozone-4, recorded in borecores GAM-3 (294-437.98 m), GAM-7 (453-640.5 m) and GAM-8 $(35.35 \mathrm{~m})$ is characterized by the dominance of Faunipollenites and sub-dominance of Striatopodocarpites along with certain stratigraphically significant taxa, viz., Weylandites, Guttulapollenites, Corisaccites, Aurangapollenites and Osmundacidites. Palynozone-5, demarcated in borecore GAM-3 (144.86-221.3 m) and borecore GAM-10 (35.35 m), is distinguished by the dominance of striate disaccates along with at least $30 \%$ pollen assigned to Striasulcites; Palynozone-6, identified in borecore GAM-3 (35.1-73.08 m) and borecore GAM-7 (231-423 m), is characterized by the dominance of striate disaccates along with $20-30 \%$ of Densipollenites; Palynozone7, recorded in borecore GAM-7 (206 m), is distinguished by the dominance of Crescentipollenites along with striate disaccates; Palynozone-8, identified in borecore GAM-7 (178.4 m), is characterized by the distinguished presence of taeniate pollen Guttulapollenites. Palynozones 4-8 belong to Raniganj Palynoflora. Palynozone-9, identified in borecore GAM-7 $(166 \mathrm{~m})$, is discriminated by the dominance of trilete spore Verrucosisporites and sub-dominance of taeniate pollen Lunatisporites which belongs to Triassic palynoflora. On the basis of palynological data Palynozone-1 (= Talchir palynoflora), Palynozone-2 and Palynozone-3 have been assigned to Early Permian age; Palynozones 4-8 (= Raniganj palynoflora) have been assigned to Late Permian age and Palynozone-9 (= Triassic palynoflora) has been assigned to Early Triassic age. With the aim of correlating the Permian and Triassic sediments of Mailaram area palynological investigations of five borecores were carried out.

Keywords. Gondwana; Godavari Graben; Talchir; Karharbari; Barakar; Raniganj; Triassic; Panchet; palynostratigraphy. 


\section{Introduction}

Palaeopalynology is a branch of palaeobotany and micropalaentology which deals with the study of plant microfossils (algae, spores, pollen, acritarchs) from the Precambrian to the Holocene. This branch is used for a diverse range of applications, related to many scientific disciplines like biostratigraphy, geochronology, palaeoecology, climate change, geothermal alteration, limnology, taxonomy, evolutionary studies, forensic palynology, allergy studies, melissopalynology and archaeological palynology. Palynology has once again become an important parameter in prospecting and economic utilization of fossil fuel exploration. Palynomorphs are extracted from the rock samples by the simple technique of maceration and are identified on the basis of their distinctive morphological characters. Well identified palynomorphs are good indicators of narrow time ranges and also helpful in the discovery of oil, coal, and gas deposits. As fossil spores and pollen are present in coal and associated sediments, these can be utilized for dating and stratigraphic correlation of coal and coal-bearing horizons.

Just as some plant groups have changed their morphological characters over time, so too their pollen and spores may have changed their organization and structure through time. Plant microfossils recovered from sediments most commonly represent the parent vegetation which was growing during the deposition of sediments. Thus, the pollen and spores in the rocks of different times are quite different in their morphological and numerical characters as a result of which the presence of particular types of pollen and spores or their groups (=Palynoassemblages) characterizes that particular horizon. Dating of coal and associated sediments is performed by the quantitative and qualitative study of these pollen and spores and correlation has been done by comparing the palynoflora of the area with the other rock strata.

Till late in the 20th century, Barakar was supposed to be the main coal bearing horizon in Godavari Graben. But with the discovery of additional younger coal horizon equivalent to Raniganj Formation, identification of additional coal horizons in newer areas is the prime driver for prospecting of coal in Godavari Graben. Inconsistency in thickness of coal seams and parting sandstones, non-consistent nature of coal piles, split of the seam into two or more units and displacement of seam due to faulting both along the strike and dip have created numerous problems in prospecting, identification, correlation and exploitation of the coal reserves in this graben, hence, the dating and correlation of coal seams is the basic requirement in this area.

The present paper is part of a comprehensive investigation intended to search and characterize the Early Permian to Early Triassic palynological succession in the Mailaram area of this graben. The main goal of this contribution is to establish a formal palynozonation in this area. Present work also validates our existing knowledge about the Permian-Triassic palynoflora of the study area.

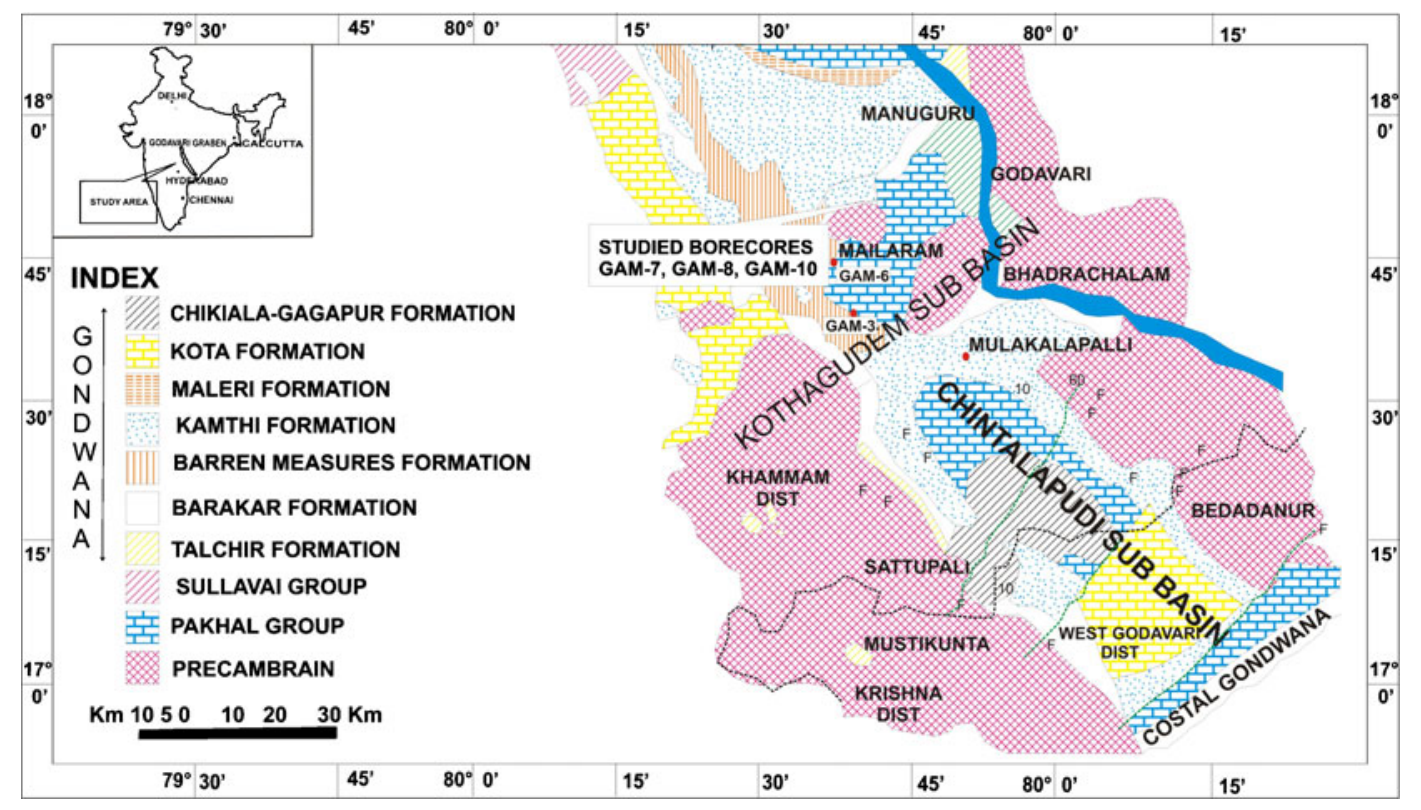

Figure 1. Location of study area and borecore samples. 


\section{Geology}

In India, the Gondwana deposits occur in peninsular as well as extra-peninsular regions. In the peninsular part, these deposits occur in a triangular pattern distributed along four major river valleys, viz., Damodar, Son-Mahanadi, Godavari and Satpura Basin. The Pranhita-Godavari (PG) Valley, a major lineament within the south Indian cratonic province, represents a continuous sequence of sedimentation during the upper Palaeozoic and Mesozoic.

Synthesis of surface and subsurface data in Mailaram area (figure 1), located between $17^{\circ} 43^{\prime} 00^{\prime \prime} \mathrm{N}$ and $80^{\circ} 37^{\prime} 05^{\prime \prime} \mathrm{E}$ in the southern part of the Godavari Graben has established the existence of Raniganj/Kamthi, Barren Measures, Barakar and Talchir sequences lying unconformably over the Precambrian rocks. The general strike of the sediments in NNW-SSE with $10^{\circ}$ to $15^{\circ}$ westerly dips. One regionally persistent coal-shale horizon of Barakar Formation ranging in thickness between 7.51 and $18.35 \mathrm{~m}$ has been recorded within 24$519 \mathrm{~m}$ depth from the surface and 176.30-226.70 m above the Talchir-Barakar formational contact. Apart from this, another coal-shale horizon belonging to lower part of Kamthi Formation has been intersected at depth of $54.11 \mathrm{~m}$ below the surface. The regionally persistent Barakar coal-shale horizon is highly interbanded with shale. The exploration at Mailaram area was taken up primarily to prove the extension of seam which is being exploited in the adjoining Manuguru area.

\section{Materials and methods}

Samples of different lithologies, viz., carbonaceous sandstone, laminated sandstone, clay, coal, grey shale, carbonaceous shale, coaly shale were processed following standard palynological method for Palaeozoic material. The techniques applied consist of the removal of silica using hydrofluoric acid (HF) for 2-3 days. The resultant demineralized residue was treated with nitric acid $\left(\mathrm{HNO}_{3}\right)$ and alkali $(10 \% \mathrm{KOH})$. Palynomorphs were concentrated, constituting final residue and slides were prepared from a few drops of final residue by mounting in Canada balsam with the help of polyvinyl chloride (PVC). The taxonomic study and the photomicrographs present in this paper, were made under Olympus BX62 microscope with DP25 camera. These slides are housed in the repository of the Birbal Sahni Institute of Palaeobotany. Yielding of the samples was good, in most of the cases at least 200 palynomorphs per sample were counted.

\section{Palynological data}

Quantitative analysis of yielding samples in five different borecores have lead to recognize three palynoassemblages in borecore GAM-3; two palynoassemblages in borecore GAM-6; six palynoassemblages in borecore GAM-7; three palynoassemblages in borecore GAM-8 and two palynoassemblages in borecore GAM-10. All taxa identified have already been described elsewhere. Selected spore and pollen species mainly of key marker taxa, from the Early Permian, Late Permian, and Early Triassic assemblages have been shown in plates 1,2 and 3 , respectively.

\subsection{Description of palynoassemblages in studied borecores}

\subsubsection{Borecore GAM-3}

Three palynoassemblages have been identified in borecore GAM-3 (Histogram-1).

Palynoassemblage-I (294-437.98 m) of this borecore shows dominance of striate disaccates chiefly Faunipollenites, Striatopodocarpites along with sub-dominance of non-striate disaccates. Frequency of different taxa in this palynoassemblage are as follows:

Triletes: Brevitriletes (1-2\%), Leiotriletes (1\%). Monosaccates: Parasaccites (2\%), Caheniasaccites $(1 \%)$.

Non-striate disaccates: Scheuringipollenites (10$32 \%)$, Ibisporites (1-4\%), Vesicaspora (4-8\%), Platysaccus (1\%).

Striate disaccates: Faunipollenites (32-46\%), Striatopodocarpites (10-30\%), Striatites (1-2\%), Lahirites (1-3\%), Striasulcites (1-3\%).

Taeniates: Guttulapollenites (1\%), Corisaccites $(2 \%)$.

Others: Weylandites (3-4\%), Latosporites (3\%), Inaperturopollenites (2-3\%), Schizosporis (1\%), Osmundacidites (1\%).

Lithostratigraphic distribution: Barakar Formation.

Palynoassemblage-II (144.86-221.3 m) shows dominance of striate disaccates chiefly Faunipollenites, Striatopodocarpites along with Striasulcites (8-22\%). Frequency of different taxa in this palynoassemblage are as follows:

Trilete: Brevitriletes (2\%).

Monosaccate: Densipollenites (1\%).

Non-striate disaccates: Scheuringipollenites (8\%), Vesicaspora (1-8\%), Platysaccus (2\%).

Striate disaccates: Faunipollenites (26-27\%), Striatopodocarpites (37-45\%), Lahirites (3\%), Distriatites $(2 \%)$ and Striasulcites (8-22\%). 

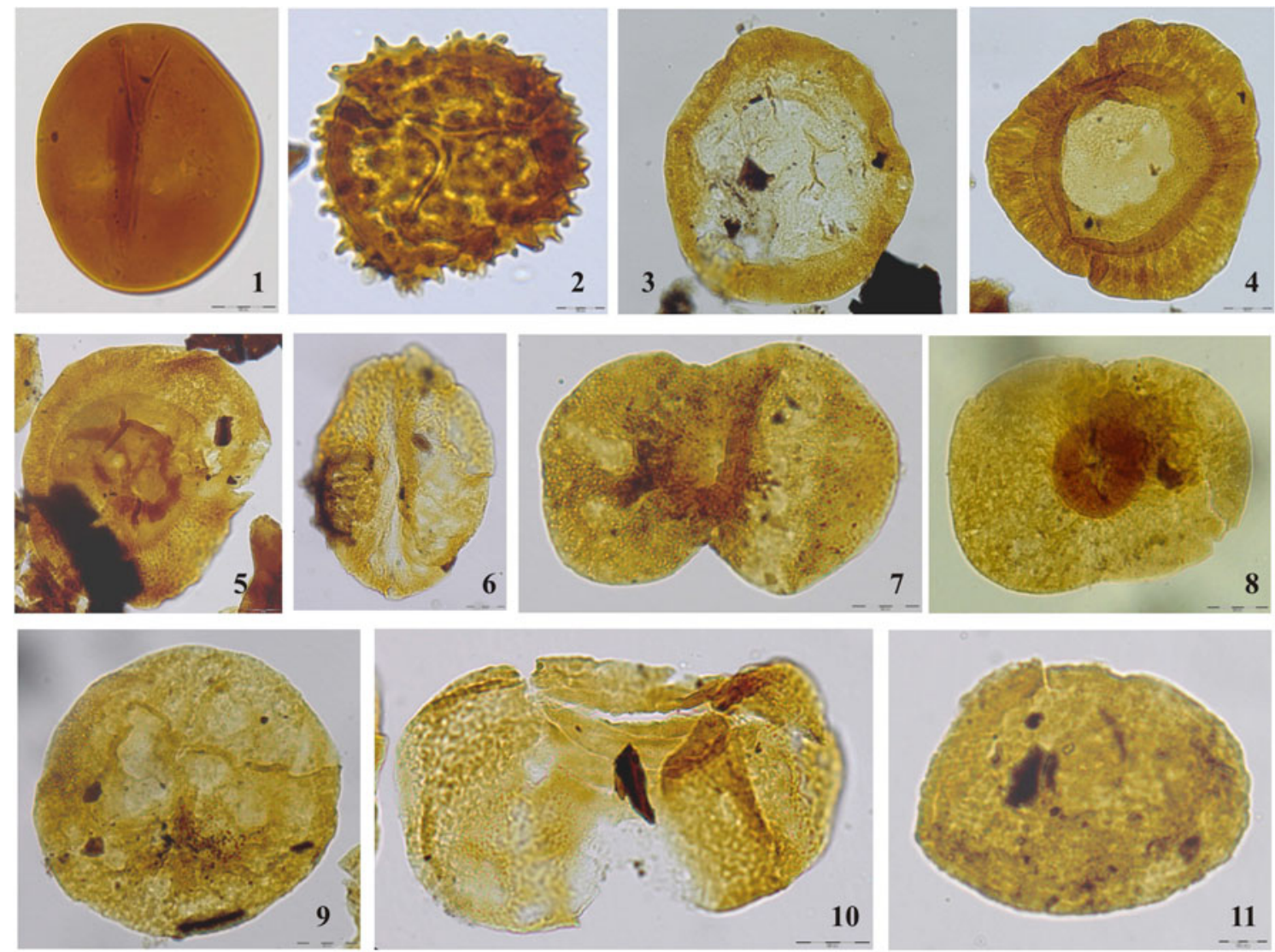

1. Callumispora barakarensis, B.S.I.P. Slide No. 10009a, U41-1 (40x), 2. Brevitriletes unicus, B.S.I.P. Slide No. 10009a, O35-2 (60x), 3. Parasaccites Korbaensis, B.S.I.P. Slide No. 10009a, W20 (40x), 4. Plicatipollenites indicus, B.S.I.P. Slide No. 10009a, O24-3 (40x), 5. Potonieisporites congoensis, B.S.I.P. Slide No. 10009a, L50-3, (40x), 6. Scheuringipollenites maximus, B.S.I.P. Slide No. 10009a, Z-47 (40x), 7. Ibisporites diplosaccus, B.S.I.P. Slide No. 10009a, T41 (40x), 8. Platysaccus densicorpus, B.S.I.P. Slide No. 10010a, K41-1 (40x), 9. Scheuringipollenites barakarensis, B.S.I.P. Slide No. 10009b, O56-3 (40x), 10. Striatopodocarpites globosus, B.S.I.P. Slide No. 10009a, S56-4 (40x), 11. Tiwariasporis simplex, B.S.I.P. Slide No. 10009b, S63-1 (40x).

Plate 1. Early Permian palynomorphs.

Taeniate: Corisaccites (2\%).

Other: Latosporites (2\%).

Lithostratigraphic distribution: Raniganj Formation.

Palynoassemblage-III (35.1-73.08 m) shows dominance of striate disaccates chiefly Faunipollenites, Striatopodocarpites along with enveloping monosaccate Densipollenites (20-32\%). Frequency of different taxa in this palynoassemblage are as follows:

Monosaccates: Densipollenites (20-32\%), Parasaccites $(2 \%)$.

Non-striate disaccates: Scheuringipollenites (6$9 \%)$, Vesicaspora (2-11\%), Vitreisporites (1-2\%). Striate disaccates: Faunipollenites (20-31\%), Striatopodocarpites (15-32\%), Striatites (1\%),
Lahirites (1-3\%), Verticipollenites (2\%) and Striasulcites (1\%).

Other: Osmundacidites (1-6\%).

Lithostratigraphic distribution: Raniganj Formation.

\subsubsection{Borecore GAM-6}

Two palynoassemblages have been identified in borecore GAM-6 (Histogram-2).

Palynoassemblage-I (331.4-500 m) of this borecore shows dominance of radial monosaccate Parasaccites (54-72\%) and sub-dominance of Plicatipollenites (2-12\%). Frequency of different taxa in this palynoassemblage are as follows:

Triletes: Brevitriletes (1\%), Verrucosisporites (1\%), Lacinitriletes (2\%), Callumispora (1-4\%), Jayantisporites (4-6\%). 

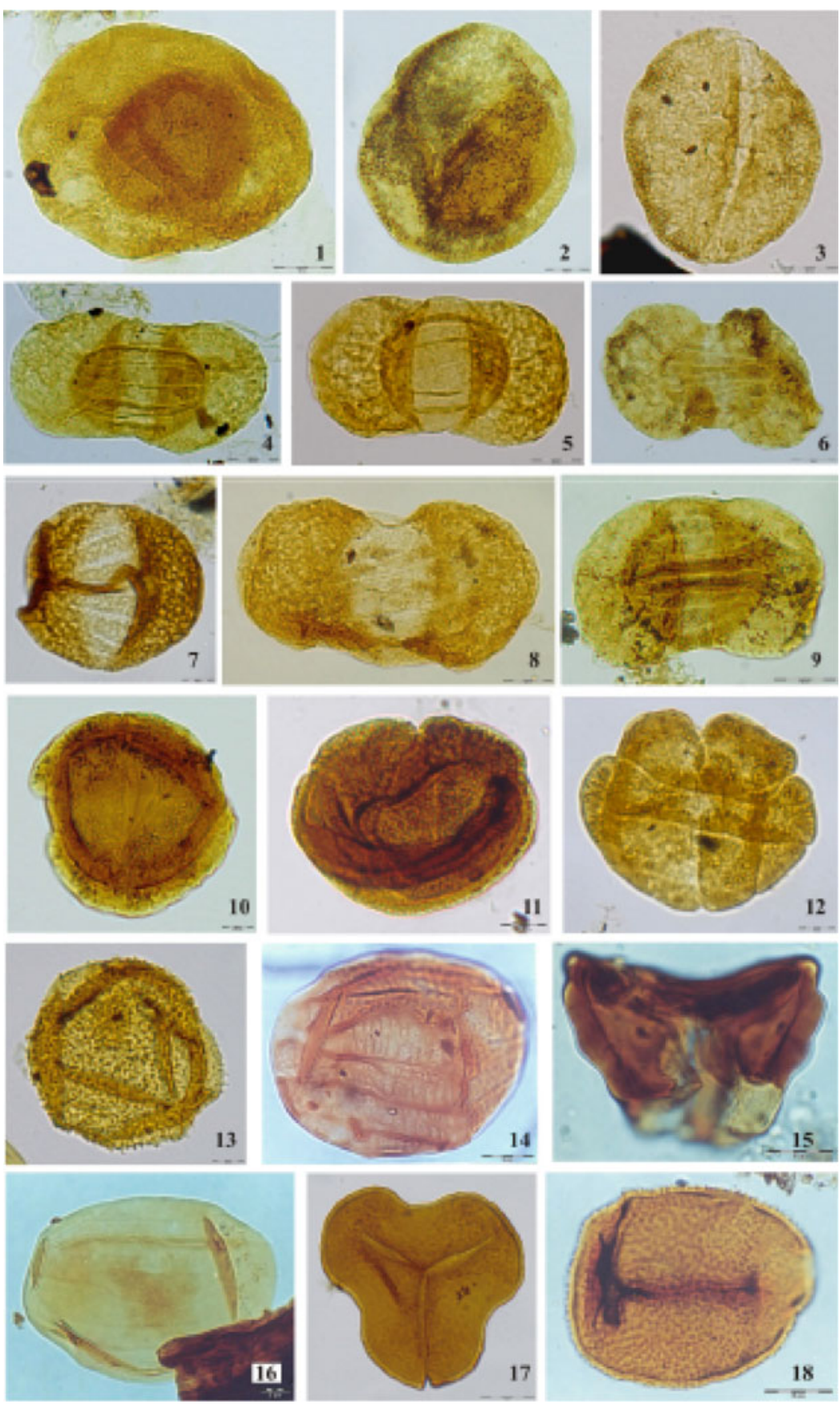

1. Densipollenites magnicorpus, B.S.I.P. Slide No. 10009,K44-3 (40x), 2. Densipollenites densus, B.S.I.P. Slide No. 10009, S69-3 (40x), 3. Scheuringipollenites maximus,B.S.I.P. Slide No. 10010, W50 (40x), 4. Crescentipollenites fuscus, B.S.I.P. Slide No. 10009, N40-1 (40x), 5. Striatopodocarpites globosus, B.S.I.P. Slide No. 10010, P47-4 (40x), 6. Striatopodocarpitessubcircularis, B.S.I.P. Slide No.10009, S59 (40x), 7. Strotersporites indicus, B.S.I.P. Slide No. 10015, F66-1 (60x), 8. Lunatisporites paliensis, B.S.I.P. Slide No. 10010b, P70-1 (40x), 9. Stroterporites crassiletus, B.S.I.P. Slide No. 10009, X68-4 (40x), 10. Corisaccites distinctus, B.S.I.P. Slide No.10009, P71-3 (40x), 11. Guttulapollenites sp., B.S.I.P. Slide No. 10010b, S35-3 (40x), 12. Guttulapollenites hannonicus, B.S.I.P. Slide No.10010b, T36-4 (60x), 13. Oumundacidites sp., B.S.I.P. Slide No. 10010, K67 (60x), 14. Columinisporites sp.,B.S.I.P. Slide No. 10009, H37 -2 (40x), 15. Iraquispora sp., B.S.I.P. Slide No. 10010, G20-2 (40x), 16. Kendosporites sp., B.S.I.P. Slide No. 10009, P51-3 (40x), 17. Phidiaesporites sp., B.S.I.P. Slide No. 10010b,H38-4 (60x), 18. Polypodiidites perverrucatus, B.S.I.P. Slide No. 10009, T40-1 (40x). 

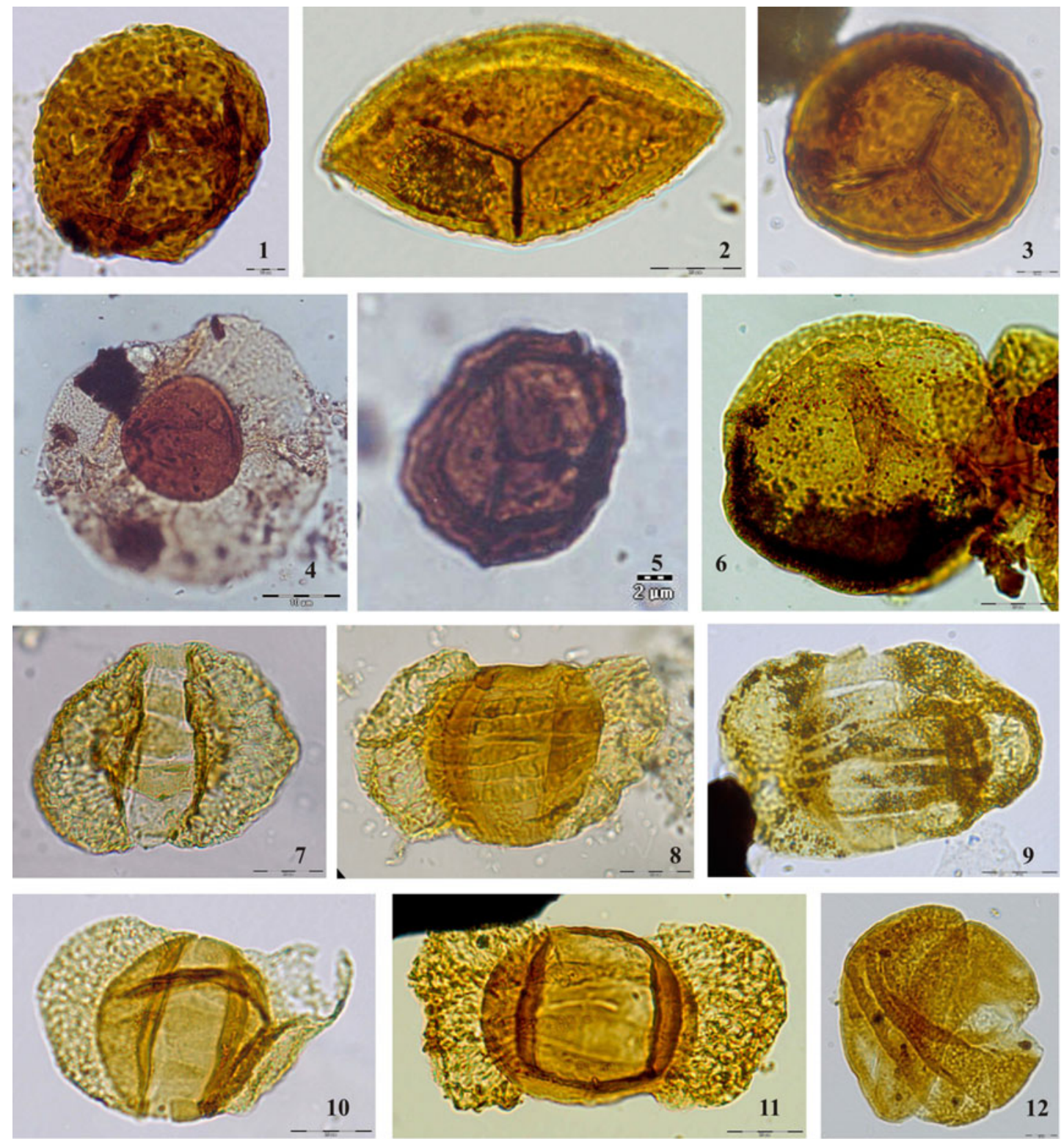

1.Verrucosisporites densus, B.S.I.P. Slide No. 10012, K49-1 (40x), 2. Verrucosisporites sp., B.S.I.P. Slide No. 10012, C60-2 (40x), 3.Guttatisporites ambiguus, B.S.I.P. Slide No. 10011, T52-2 (60x), 4. Playfordiaspora cancellosa, B.S.I.P. Slide No. 10013, O37-2 (40x), 5. Ringosporites ringus, B.S.I.P. Slide No. 10012, H49-1 (40x), 6. Lundbladispora sp., B.S.I.P. Slide No. 10011, F41-1 (40x), 7. Lunatisporites pellucidus, 10013, Q67-2 (40x), 8. Lunatisporites diffusus, B.S.I.P. Slide No. 10013, Q51 (40x), 9. Lunatisporites ovatus, B.S.I.P. Slide No. 10012, N45-2 (60x), 10. Strotersporites decorus, B.S.I.P. Slide No. 10011, Q46-2 (40x), 11. Taeniaepollenites sp., B.S.I.P. Slide No. 10012, W65-4 (40x), 12. Guttulapollenites hannonicus, B.S.I.P. Slide No. 10011, K54 (40x).

Plate 3. Early Triassic palynomorphs.

Monosaccates: Parasaccites (54-72\%), Plicatipollenites (2-12\%), Caheniasaccites (3-9\%), Virkkipollenites (2\%), Potonieisporites (1\%).

Non-striate disaccates: Scheuringipollenites (1$2 \%)$, Vesicaspora (2\%), Vestigisporites (2\%), Sahnites (2\%).

Striate disaccates: Striatopodocarpites (2\%), Faunipollenites (1\%).
Others: Plicatisporites (6\%), Leiosphaeridia (2$8 \%$ ).

Lithostratigraphic distribution: Barakar Formation.

Palynoassemblage-II (149.7-240.5 m) shows dominance of non-striate disaccates chiefly Scheuringipollenites (32-52\%) and sub-dominance 

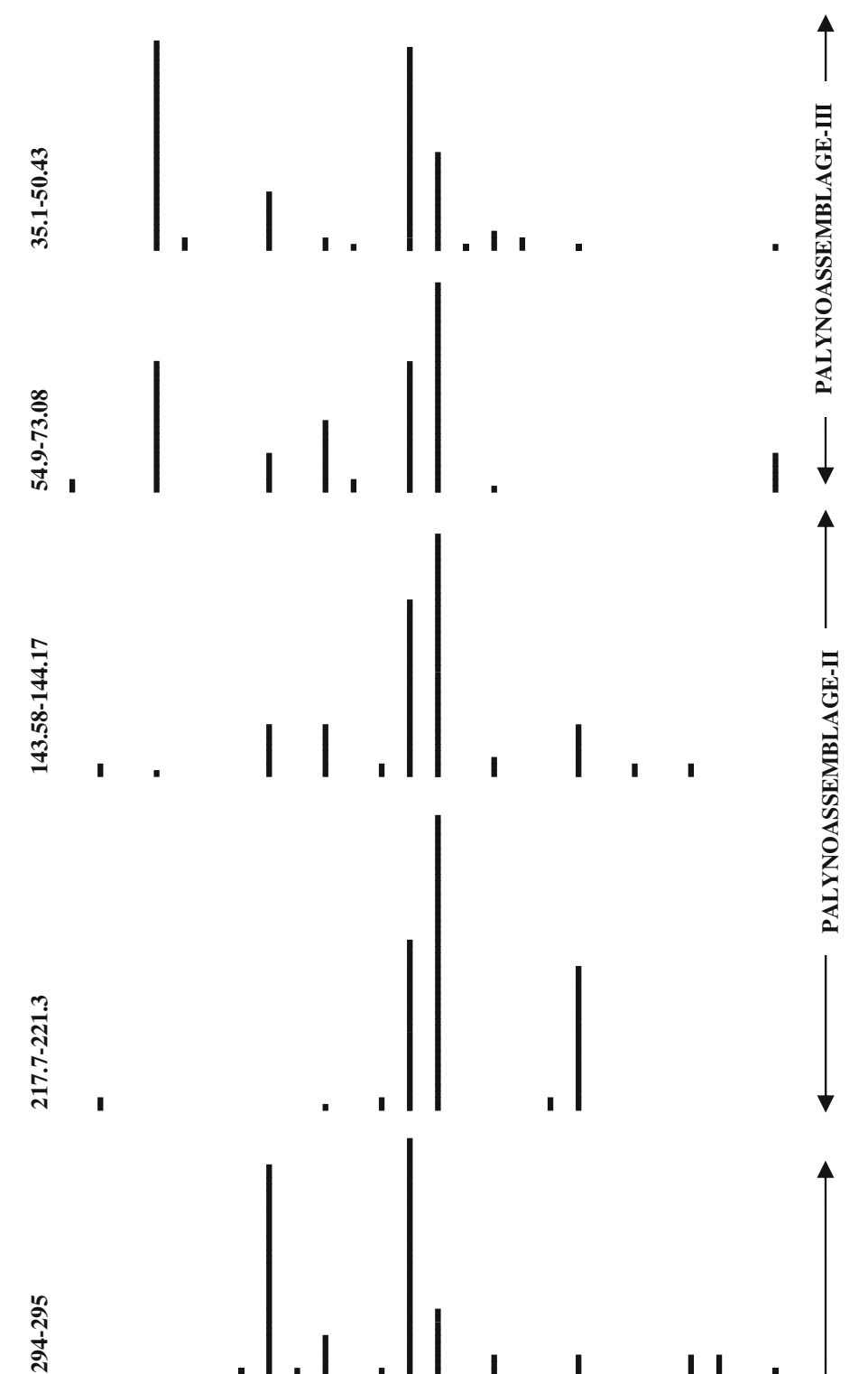


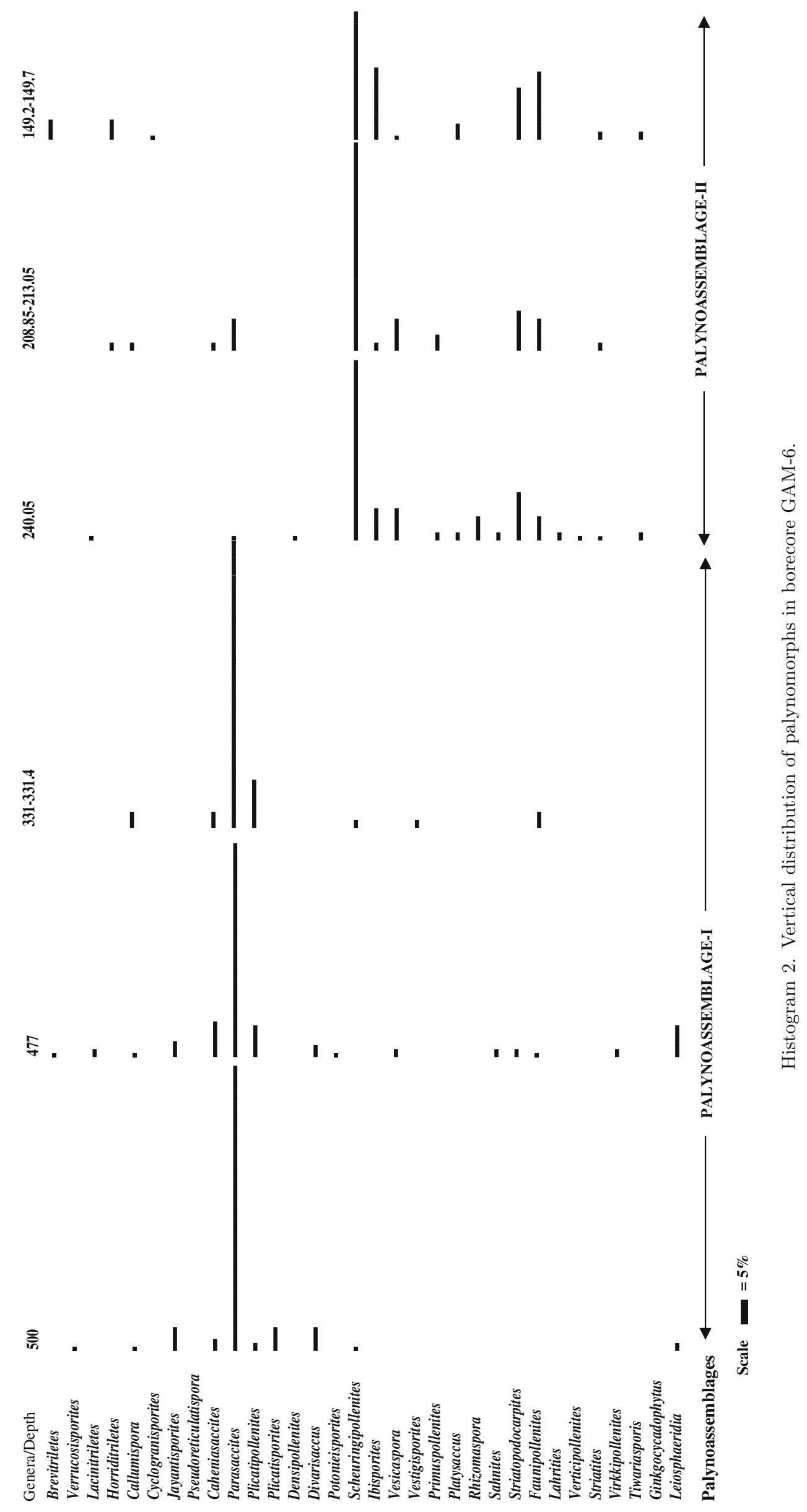




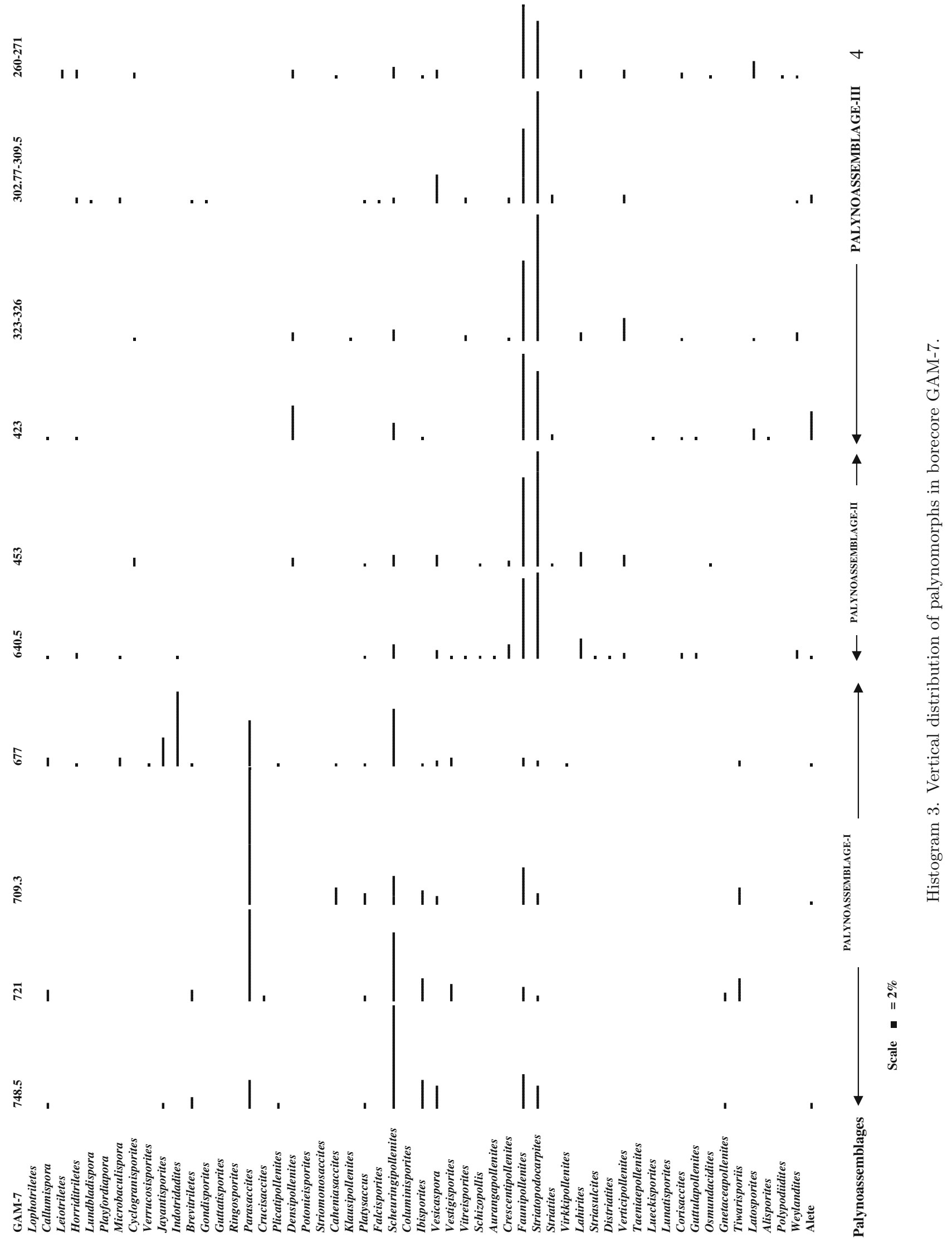




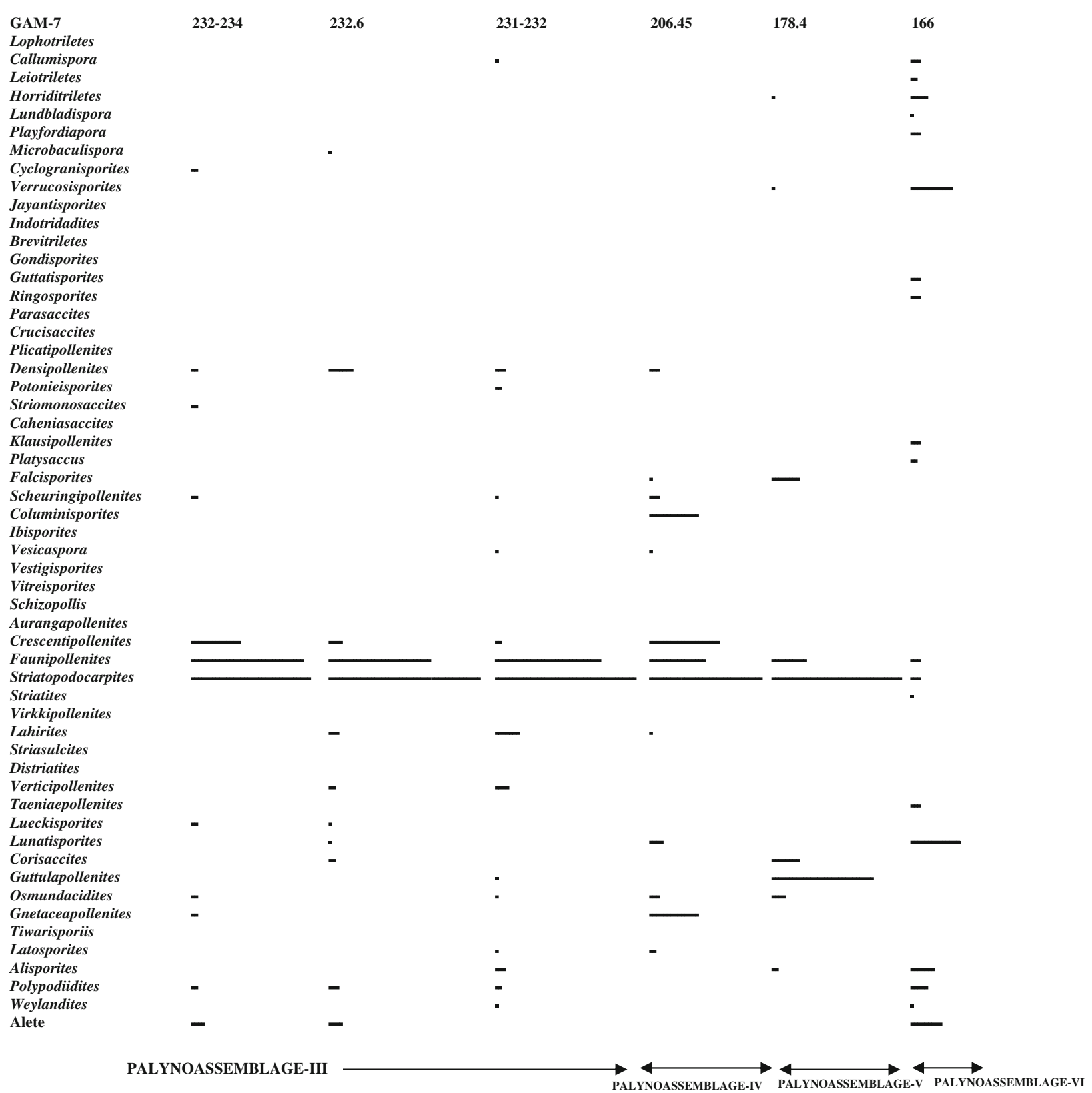

Histogram 3. (Continued)

of striate disaccates. Frequency of different taxa in this palynoassemblage are as follows:

Triletes: Brevitriletes (5\%), Lacinitriletes (1\%), Horriditriletes (2-5\%), Callumispora (1-4\%), Cyclogranisporites (1\%).

Monosaccates: Parasaccites (1-8\%), Caheniasaccites (2\%), Densipollenites (1\%).

Non-striate disaccates: Scheuringipollenites (32$52 \%)$, Ibisporites (2-18\%), Vesicaspora (1-8\%), Rhizomaspora (6\%), Primuspollenites (2-4\%), Platysaccus (2-4\%), Sahnites (2\%).

Striate disaccates: Striatopodocarpites (10-13\%), Faunipollenites (6-17\%), Lahrities (2\%), Verticipollenites (1\%) and Striatites (1-2\%).
Other: Tiwariasporis (2\%).

Lithostratigraphic distribution: Barakar Formation.

\subsubsection{Borecore GAM-7}

Six palynoassemblages have been identified in borecore GAM-7 (Histogram-3).

Palynoassemblage-I (667-749 m) of this borecore shows dominance of Parasaccites (10$48 \%$ ) and sub-dominance of Scheuringipollenites $(10-36 \%)$. Frequency of different taxa in this palynoassemblage are as follows:

Triletes: Jayantisporites (2-10\%), Indotriradites (26\%), Lophotriletes (1\%), Callumispora (2-4\%), 
Horriditriletes (1\%), Microbaculispora (3\%), Verrucosisporites (1\%), Brevitriletes (1-4\%).

Monosaccates: Parasaccites (10-48\%), Crucisaccites (2\%), Plicatipollenites (1-2\%), Virkkipollenites $(1 \%)$.

Non-striate disaccates: Scheuringipollenites (10$36 \%)$, Ibisporites (1-10\%), Vesicaspora (2-8\%), Platysaccus (1-4\%), Vestigisporites (3-6\%).

Striate disaccates: Faunipollenites (3-14\%), Striatopodocarpites (2-8\%).

Others: Gnetaceapollenites (2-3\%), Tiwariasporis (2-8\%), Inaperturopollenites (1-2\%).

Lithostratigraphic distribution: Barakar Formation.

Palynoassemblage-II (453-640.5 m) shows dominance of striate disaccates, viz., Striatopodocarpites (30-40\%) and Faunipollenites $(28-31 \%)$ and sub-dominance of non-striate disaccates. Frequency of different taxa in this palynoassemblage are as follows:

Triletes: Callumispora (1\%), Horriditriletes (2\%), Microbaculispora (1\%), Cyclogranisporites (3\%), Indotriradites (1\%).

Monosaccate: Densipollenites (3\%).

Non-striate disaccates: Scheuringipollenites (4$5 \%$, Platysaccus (1\%), Vesicaspora (3-4\%), Vestigisporites (1\%), Vitreisporites (1\%), Schizopollis (1\%), Aurangapollenites (1\%).

Striate disaccates: Faunipollenites (28-31\%), Striatopodocarpites (30-40\%), Lahirites (5-7\%), Crescentipollenites (2-5\%), Striatites (1\%), Striasulcites (1\%), Distriatites (1\%), Verticipollenites $(2 \%)$.

Taeniates: Corisaccites (2\%), Guttulapollenites (2\%). Others: Inaperturopollenites (1\%), Weylandites (3\%). Lithostratigraphic distribution: Barren Measures Formation.

Palynoassemblage-III (231-423 m) shows dominance of striate disaccates chiefly Faunipollenites, Striatopodocarpites along with Densipollenites (3-12\%). Frequency of different taxa in this palynoassemblage are as follows:

Triletes: Lophotriletes (13-5\%), Callumispora (1\%), Leiotriletes (3\%), Horriditriletes (1-3), Microbaculispora (1-2\%), Cyclogranisporites (1$2 \%$ ), Brevitriletes (1\%), Gondisporites (1\%), Lundbldispora (1\%).

Monosaccates: Densipollenites (3-12\%), Potonieisporites $(2 \%)$, Striomonosaccites $(2 \%)$, Caheniasaccites $(1 \%)$.

Non-striate disaccates: Klausipollenites (1\%), Platysaccus (1\%), Scheuringipollenites (1-6\%), Ibisporites (1\%), Vesicaspora (3-10\%), Vitreisporites (2\%), Schizopollis (1\%), Falcisporites (1\%). Striate disaccates: Crescentipollenites (1-7\%), Faunipollenites (26-36\%), Striatopodocarpites
(20-44\%), Striatites (2-3\%), Lahirites (3-7\%), Verticipollenites (2-8\%).

Taeniates: Lueckisporites (1-2\%), Corisaccites (1$2 \%$ ), Guttulapollenites (1\%), Lunatisporites (1\%). Others: Osmundacidites (1-2\%), Gnetaceapollenites (2\%), Latosporites (1-6\%), Alisporites (1$3 \%)$, Polypodiidites (1-4\%), Weylandites (1-3\%), Kendosporites (1\%), Aletes (4-10\%).

Lithostratigraphic distribution: Raniganj Formation.

Palynoassemblage-IV (206 m) shows dominance of striate disaccates chiefly Striatopodocarpites, Faunipollenites along with Crescentipollenites (20\%). Frequency of different taxa in this palynoassemblage are as follows:

Monosaccate: Densipollenites (3\%).

Non-striate disaccates: Scheuringipollenites (3\%), Columinisporites (14\%), Falcisporites (1\%), Vesicaspora (1\%).

Striate disaccates: Striatopodocarpites (32\%), Crescentipollenites (20\%), Faunipollenites (16\%), Strotersporites (1\%), Lahirites (1\%).

Taeniate: Lunatisporites (4\%).

Others: Osmundacidites (3\%), Gnetaceapollenites (14\%), Latosporites (2\%), Kendosporites (4\%).

Lithostratigraphic distribution: Raniganj Formation.

Palynoassemblage-V (178.4 m) shows dominance of striate disaccates chiefly Striatopodocarpites, Faunipollenites along with taeniate pollen Guttulapollenites (29\%). Frequency of different taxa in this palynoassemblage are as follows:

Triletes: Horriditriletes (1\%), Verrucosisporites $(1 \%)$.

Non-striate disaccate: Falcisporites (8\%).

Striate disaccates: Striatopodocarpites (37\%), Faunipollenites (10\%).

Taeniates: Corisaccites (8\%), Guttulapollenites $(29 \%)$.

Others: Osmundacidites (4\%), Alisporites (2\%), Phidiaesporites (1\%), Triquitrites (1\%), Iraquispora (1\%), Cyathidites (1\%), Concavissimisporites $(1 \%)$.

Lithostratigraphic distribution: Raniganj Formation.

Palynoassemblage-VI (166 m) shows dominance of trilete spore Verrucosisporites (12\%) and taeniate Lunatisporites (32\%) along with some stratigraphically significant taxa, viz., Lundbladispora (1\%), Playfordiaspora (3\%), Guttatisporites (3\%), Ringosporites (4\%). Frequency of different taxa in this palynoassemblage are as follows:

Triletes: Verrucosisporites (12\%), Horriditriletes (6\%), Callumispora (4\%), Leiotriletes (2\%), Lundbladispora (1\%), Playfordiaspora (3\%), Guttatisporites (3\%), Ringosporites (4\%). 
Non-striate disaccate: Klausipollenites (4\%) Platysaccus (2\%).

Striate disaccates: Faunipollenites (4\%), Striatopodocarpites (4\%), Strotersporites (3\%), Striatites (1\%), Taeniaepollenites $(3 \%)$.

Taeniate: Lunatisporites (32\%).

Others: Alisporites (8\%), Polypodiidites (6\%), Weylandites (1\%) and Alete (10\%).

Lithostratigraphic distribution: Raniganj Formation.

\subsubsection{Borecore GAM-8}

Three palynoassemblages have been identified in borecore GAM-8 (Histogram-4).

Palynoassemblage-I $(89.75 \mathrm{~m})$ of this borecore shows dominance of Parasaccites and subdominance of Scheuringipollenites. Frequency of different taxa in this palynoassemblage are as follows:

Triletes: Brevitriletes (4\%), Callumispora (2\%), Apiculatisporites (2\%), Leiotriletes (1\%).
Monosaccates: Parasaccites (30\%), Plicatipollenites (8\%), Virkkipollenites (4\%), Caheniasaccites (2\%), Potonieisporites (1\%).

Non-striate disaccates: Scheuringipollenites (17\%), Primuspollenites (12\%), Vesicaspora (1\%), Vestigisporites (1\%).

Striate disaccates: Faunipollenites (1\%), Striatites $(1 \%)$.

Others: Latosporites (4\%), Leiosphaeridia (1\%).

Lithostratigraphic distribution: Barakar Formation.

Palynoassemblage-II (84.95 m) shows dominance of non-striate disaccates Scheuringipollenites (24\%). Frequency of different taxa in this palynoassemblage are as follows:

Triletes: Brevitriletes (10\%), Horriditriletes (8\%), Lacinitriletes (2\%), Apiculatisporites (9\%).

Non-striate disaccates: Scheuringipollenites (24\%), Ibisporites (5\%), Primuspollenites (3\%), Platysaccus (3\%), Vesicaspora (2\%).

Striate disaccates: Faunipollenites (8\%), Striatopodocarpites (3\%), Striatites (1\%).

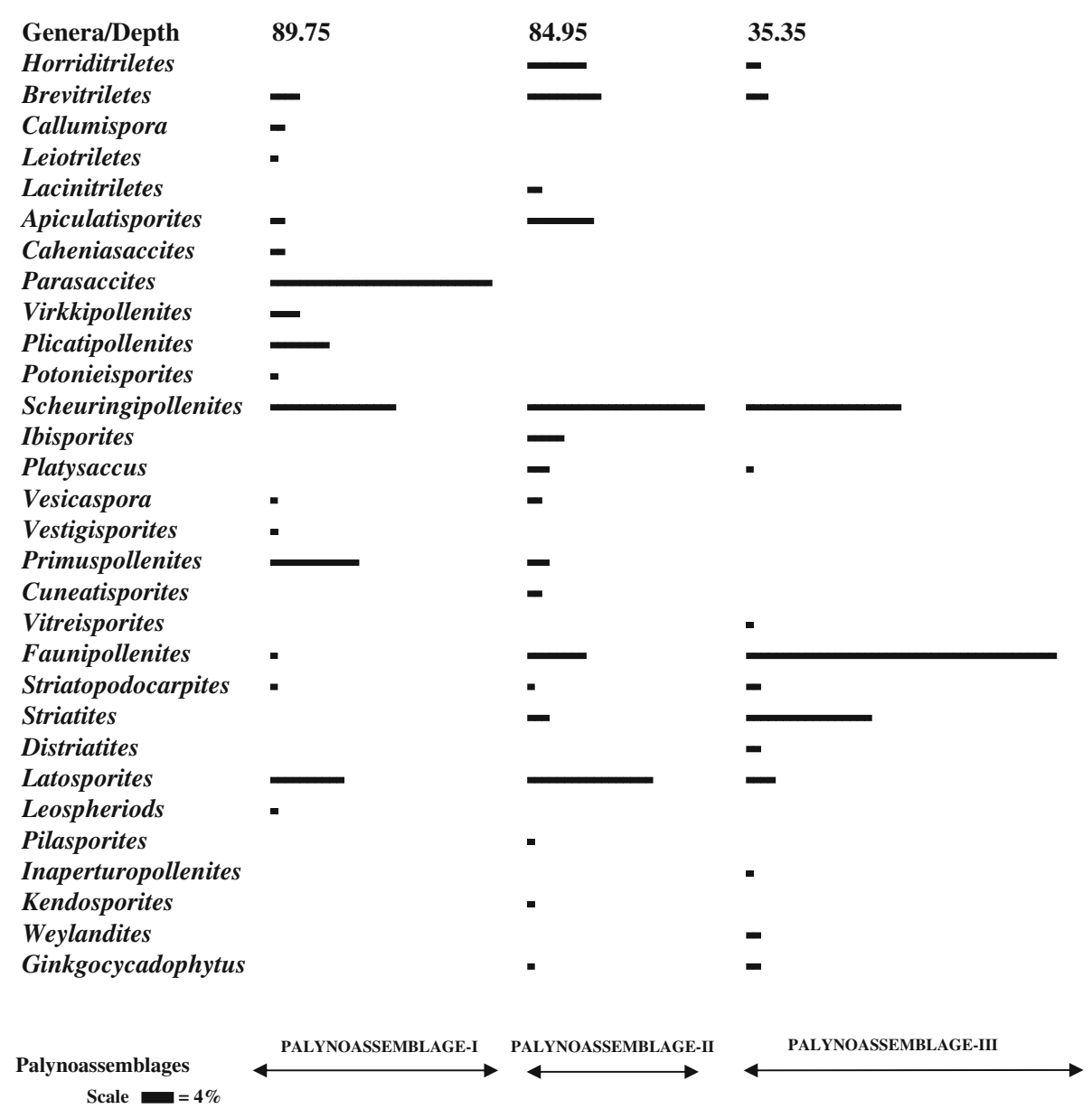

Histogram 4. Vertical distribution of palynomorphs in borecore GAM-8. 
Others: Latosporites (17\%), Pilasporites (1\%), Inaperturopollenites (1\%), Kendosporites (1\%), Weylandites (2\%), Ginkgocycadophytus (2\%).

Lithostratigraphic distribution: Barakar Formation.

Palynoassemblage-III (35.35 m) shows dominance of striate disaccates Faunipollenites (42\%), Striatopodocarpites (17\%) and sub-dominance of non-striate disaccate Scheuringipollenites (21\%). Frequency of different taxa in this palynoassemblage are as follows:

Triletes: Horriditriletes (2\%), Brevitriletes (3\%).

Non-striate disaccates: Scheuringipollenites (21\%), Platysaccus (1\%), Vitreisporites (1\%).

Striate disaccates: Faunipollenites (42\%), Striatopodocarpites (17\%), Striatites (2\%), Distriatites $(2 \%)$.

Others: Latosporites (4\%), Weylandites (2\%), Ginkgocycadophytus (2\%), Inaperturopollenites $(1 \%)$.

Lithostratigraphic distribution: Raniganj Formation.

\subsubsection{Borecore GAM-10}

Two palynoassemblages have been identified in borecore GAM-10 (Histogram-5).

Palynoassemblage-I (505.66-581.55 m) of this borecore shows dominance of monosaccate Parasaccites (42-80\%) along with other monosaccates. Frequency of different taxa in this palynoassemblage are as follows:

Triletes: Brevitriletes (2\%), Callumispora (4-10\%), Calamospora (4\%), Jayantisporites (2-5\%).

Monosaccates: Parasaccites (42-80\%), Caheniasaccites (6-10\%), Divarisaccus (2-10\%), Plicatipollenites (6-8\%), Potonieisporites (1\%), Crucisaccites $(1 \%)$.

Non-striate disaccates: Scheuringipollenites (6\%), Vesicaspora (6\%), Sahnites (4\%), Vestigisporites (2\%), Platysaccus (2\%).

Striate disaccates: Faunipollenites (2-4\%), Striatopodocarpites (1\%).

Others: Ginkgocycadophytus (1\%), Pilasporites $(1 \%)$.

Lithostratigraphic distribution: Talchir Formation.

Palynoassemblage-II $(61.5 \mathrm{~m})$ of this borecore shows dominance of striate disaccates along with Straisulcites (30\%). Frequency of different taxa in this palynoassemblage are as follows:

Monosaccates: Parasaccites (1\%), Densipollenites $(1 \%)$.

Non-striate disaccates: Schizopollis (7\%), Platysaccus (2\%), Vesicaspora (1\%).
Striate disaccates: Faunipollenites (32\%), Striatopodocarpites (12\%), Verticipollenites (2\%), Striatites (1\%), Striasulcites (30\%), Circumstriatites $(2 \%)$.

Others: Inaperturopollenites (7\%), Osmundacidites (1\%), Schizosporis (1\%).

Lithostratigraphic distribution: Barakar Formation.

\subsection{Palynozonation}

The palynozonations proposed here is based on the morphographic study of the spore and pollen grains, recovered from the Early Permian to Early Triassic succession of the Mailaram area (figure 2). Taking into account the generic dominance and distributional patterns of various spore-pollen taxa, as well as stratigraphically significant spore-pollen taxa, the nine assemblage zones, described here, have been identified belonging to Talchir, Karharbari, Barakar, Raniganj and Panchet palynoflora from five borecores of the study area. Major composition of the assemblage zones have been written with each assemblage zone while the complete listing of the species in the particular assemblage zone has been represented in table 1 .

\subsubsection{Palynozone-1}

Representative Occurrences

Palynozone-1 includes:

- Palynoassemblage-I of borecore GAM-6

- Palynoassemblage-I of borecore GAM-10

Composition: Dominance of monosaccates, viz., Parasaccites, Plicatipollenites and Caheniasaccites along with very few non-striate and striate disaccates in these two palynoassemblages signifies Talchir palynoflora (Early Permian) to this palynozone. This palynozone is characterized by the presence of Parasaccites korbaensis, P. monoletes, $P$. obscurus, $P$. bilateralis, $P$. longus, $P$. talchirensis along with Plicatipollenites diffuses, P. gondwanensis, $P$. indicus, Caheniasaccites elongatus, $C$. ellipticus. Besides these monosaccates, presence of Jayantisporites pseudozonatus, Divarisaccus lelei and Sahnites jayantiensis makes this palynozone significant.

Age span: Asselian-Sakmarian age (Jha 2006)

Remarks: This palynozone corresponds Parasaccites korbaensis assemblage zone of Tiwari and Tripathi (1992) from Damodar Basin. 


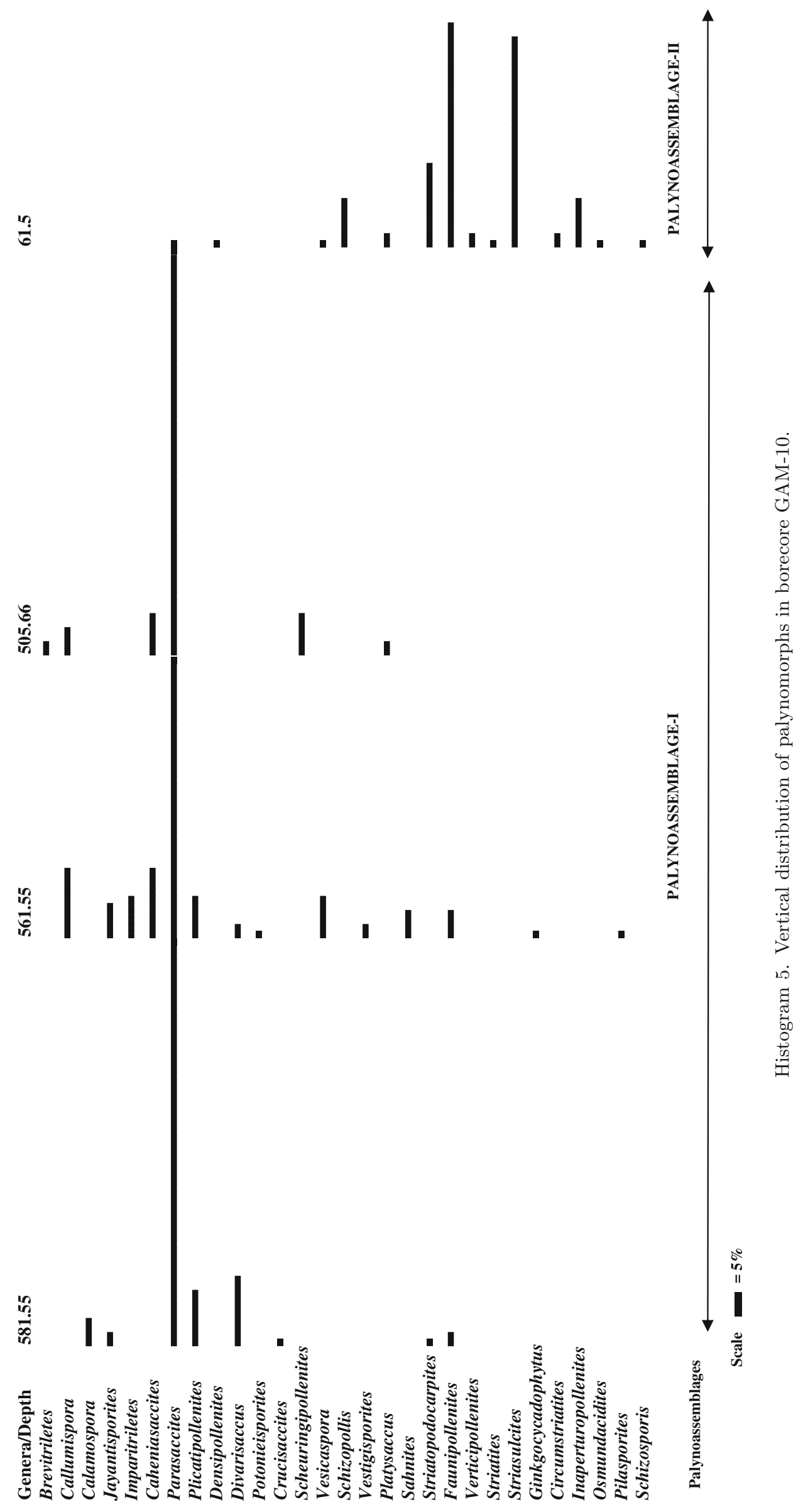




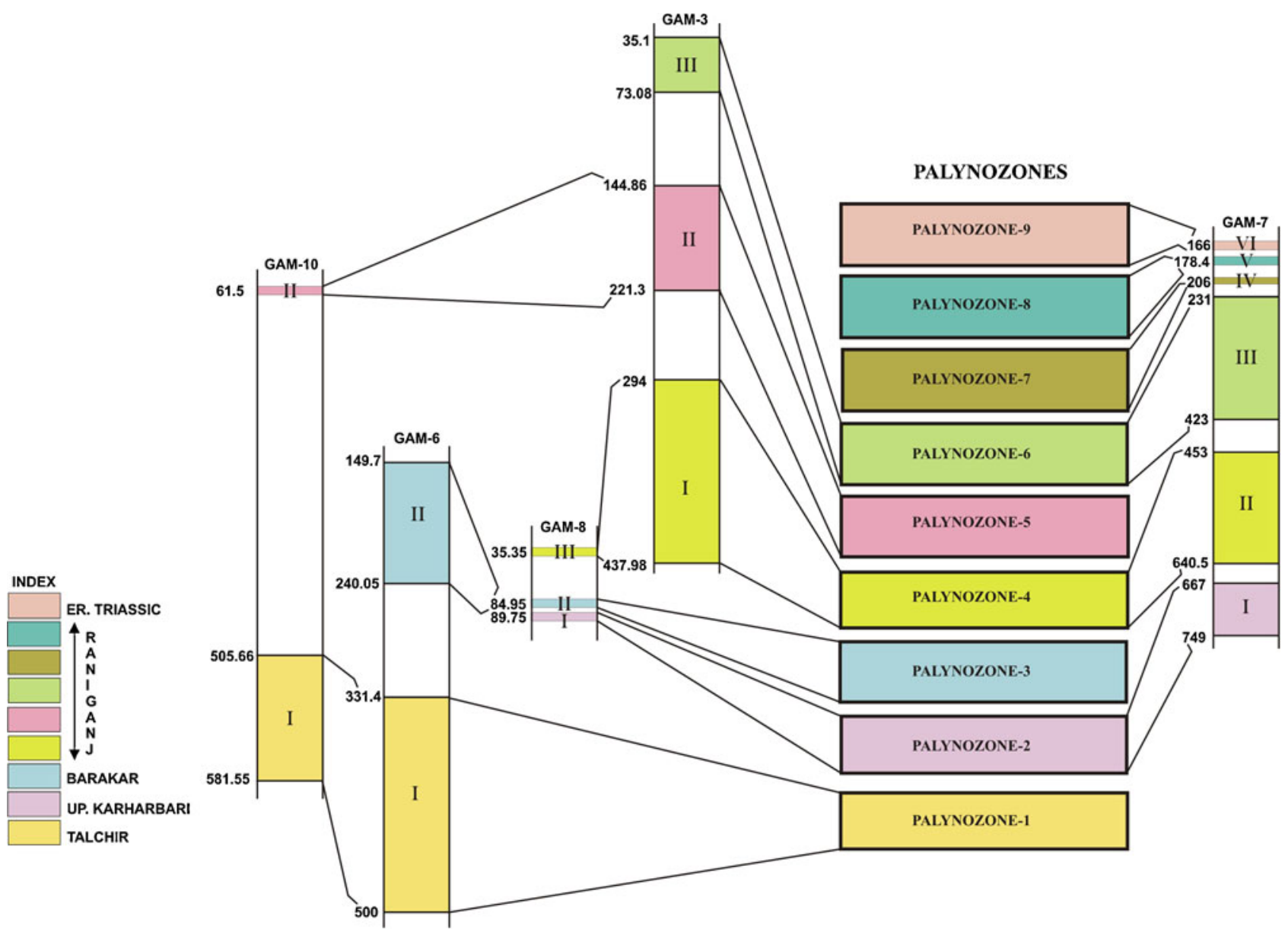

Figure 2. Correlation of studied borecores of Mailaram area.

\subsubsection{Palynozone-2}

Representative Occurrences

Palynozone-2 includes:

- Palynoassemblage-I of borecore GAM-7

- Palynoassemblage-I of borecore GAM-8

Composition: Palynozone-2 is distinguished by dominance of monosaccate Parasaccites, subdominance of non-striate disaccate Scheuringipollenites along with 2-26\% of triletes, viz., Indotriradites, Jayantisporites and very few striate disaccates. Presence of Parasaccites korbaensis, P. distinctus, P. diffuses, P. obscures, Scheuringipollenites barakarensis, $S$. maximus, $S$. tentulus, Ibisporites jhingurdahiensis, Caheniasaccites elongates, C. distinctus, C. ellipticus, C. ovatus, Plicatipollenites indicus, Potonieisporites neglectus, Crucisaccites indicus, Callumispora barakarensis. First appearence of Parasaccites distinctus, $P$. diffuses, Caheniasaccites ovatus, C. distinctus, Potonieisporites neglectus, Crucisaccites indicus, Scheuringipollenites barakarensis, S. tentulus, Striatopodocarpites diffuses, S. decorus, Faunipollenites parvus makes it different from Palynozone-1.
Composition of this palynozone represents Upper Karharbari (Early Permian) palynoflora.

Age span: Artinskian age (Jha 2006).

Remarks: This palynozone has not been identified in Damodar Basin (Tiwari and Tripathi 1992).

\subsubsection{Palynozone-3}

Representative Occurrences

Palynozone-3 includes:

- Palynoassemblage-II of borecore GAM-6

- Palynoassemblage-II of borecore GAM-8

Composition: Dominance of non-striate disaccates, viz., Scheuringipollenites barakarensis, $S$. maximus, S. tentulus, Ibisporites jhingurdahiensis, Platysaccus densicorpus, P. leschiki, P. papillionis, P. plicatus, Primuspollenites levis and subdominance of striate disaccates, viz., Faunipollenites gopadensis, F. goraiensis, Striatopodocarpites decorus, S. globosus, S. labrus, S. rotundus, $S$. 


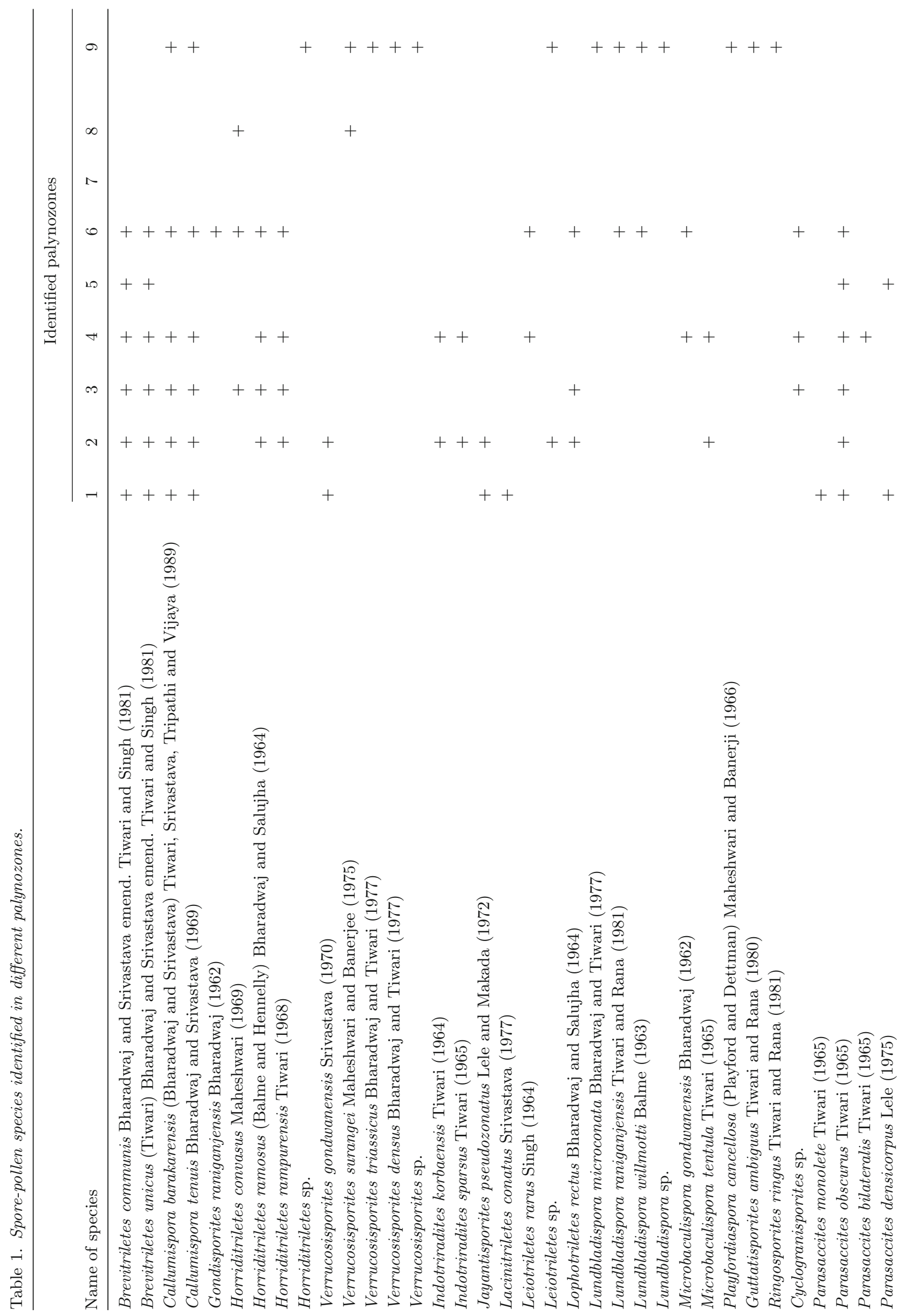




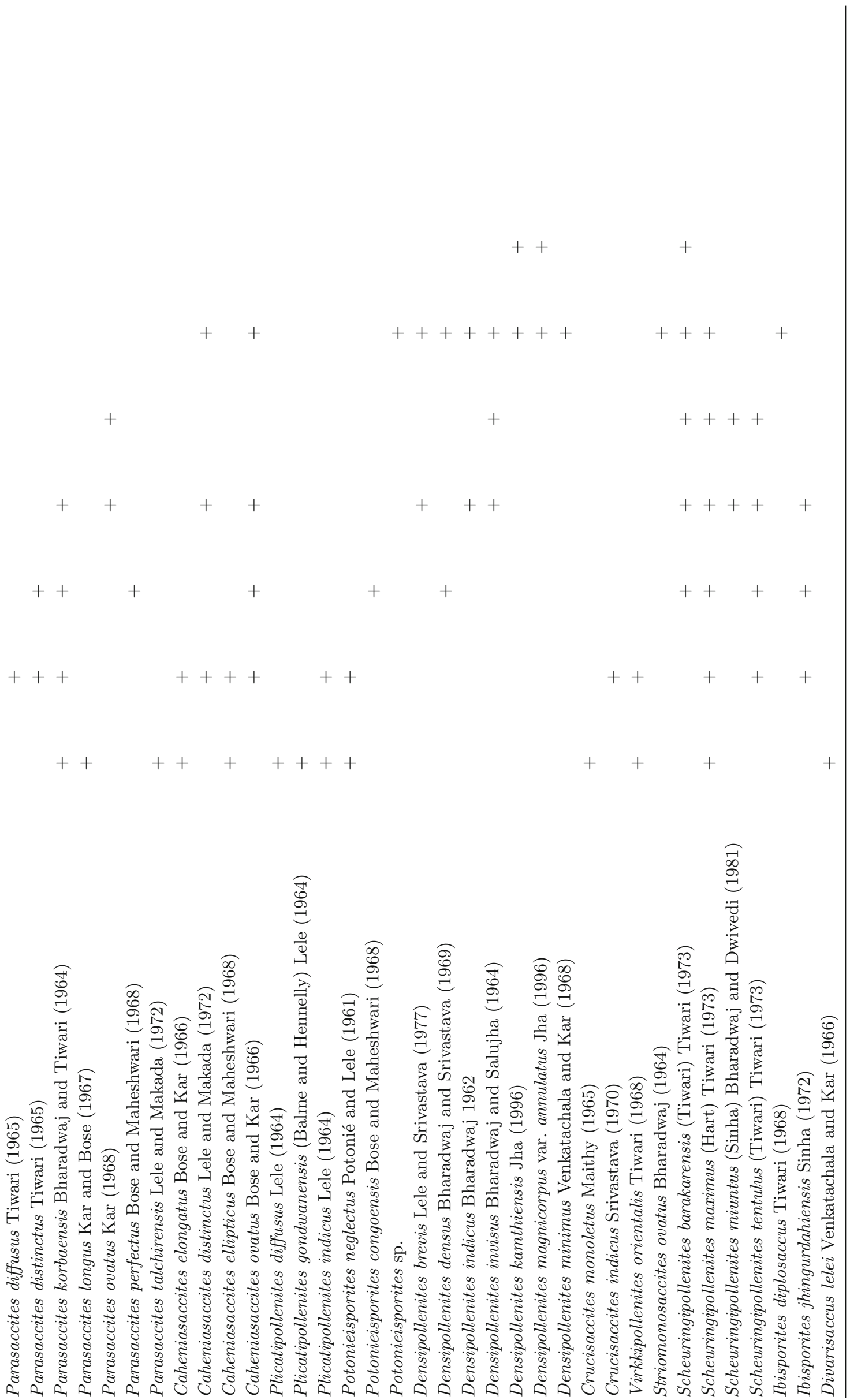




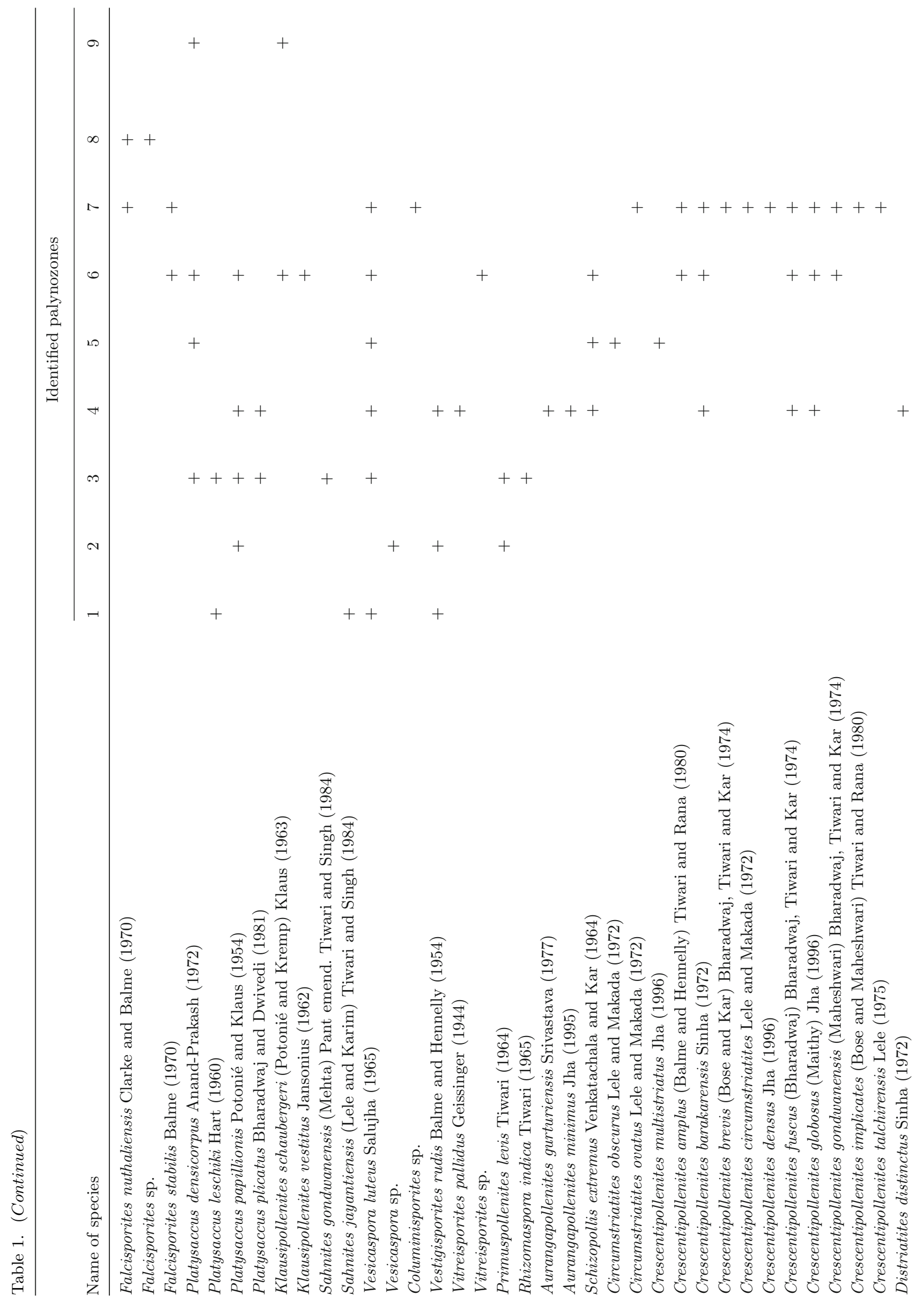


Permian-Triassic palynostratigraphy in Mailaram area

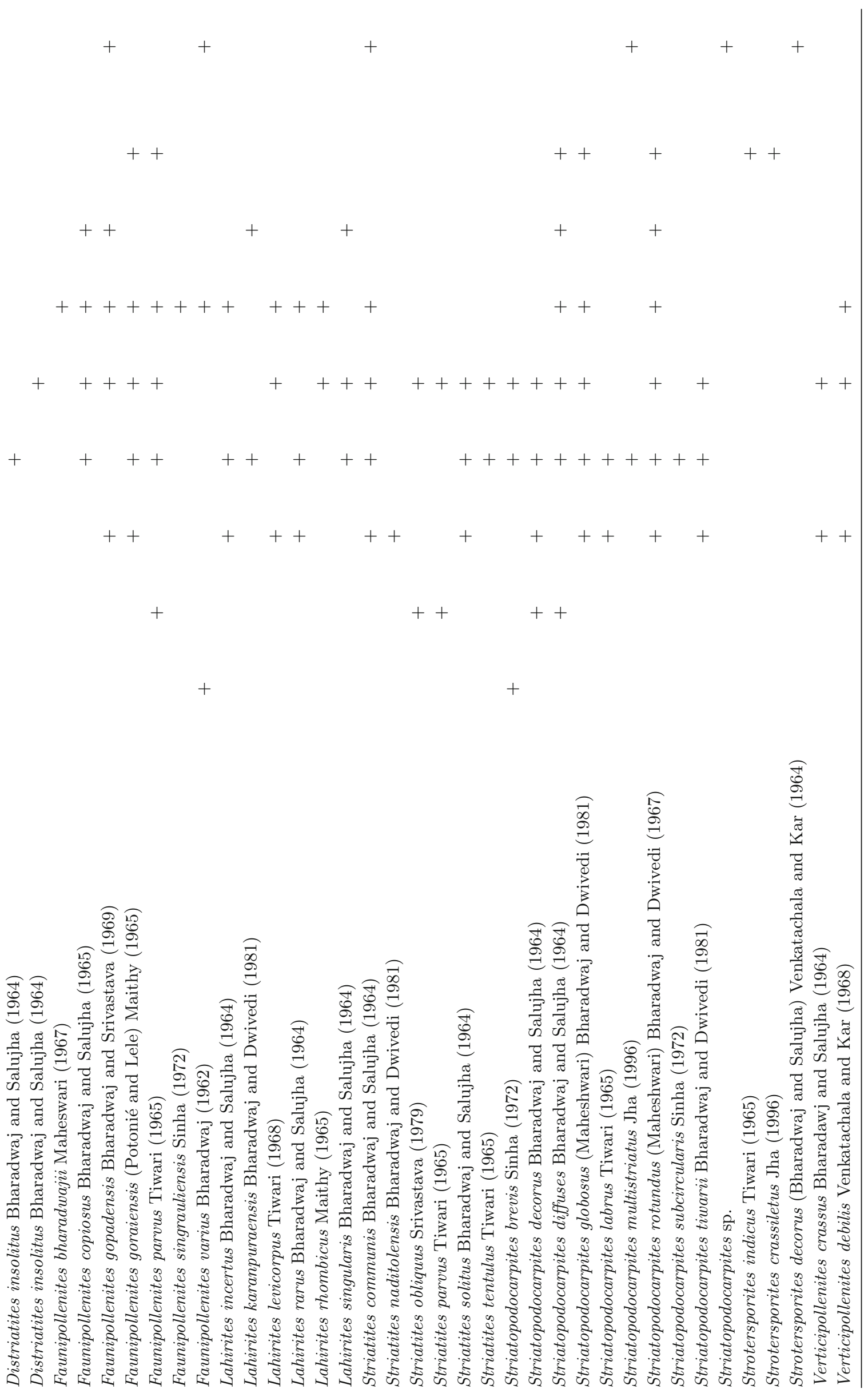




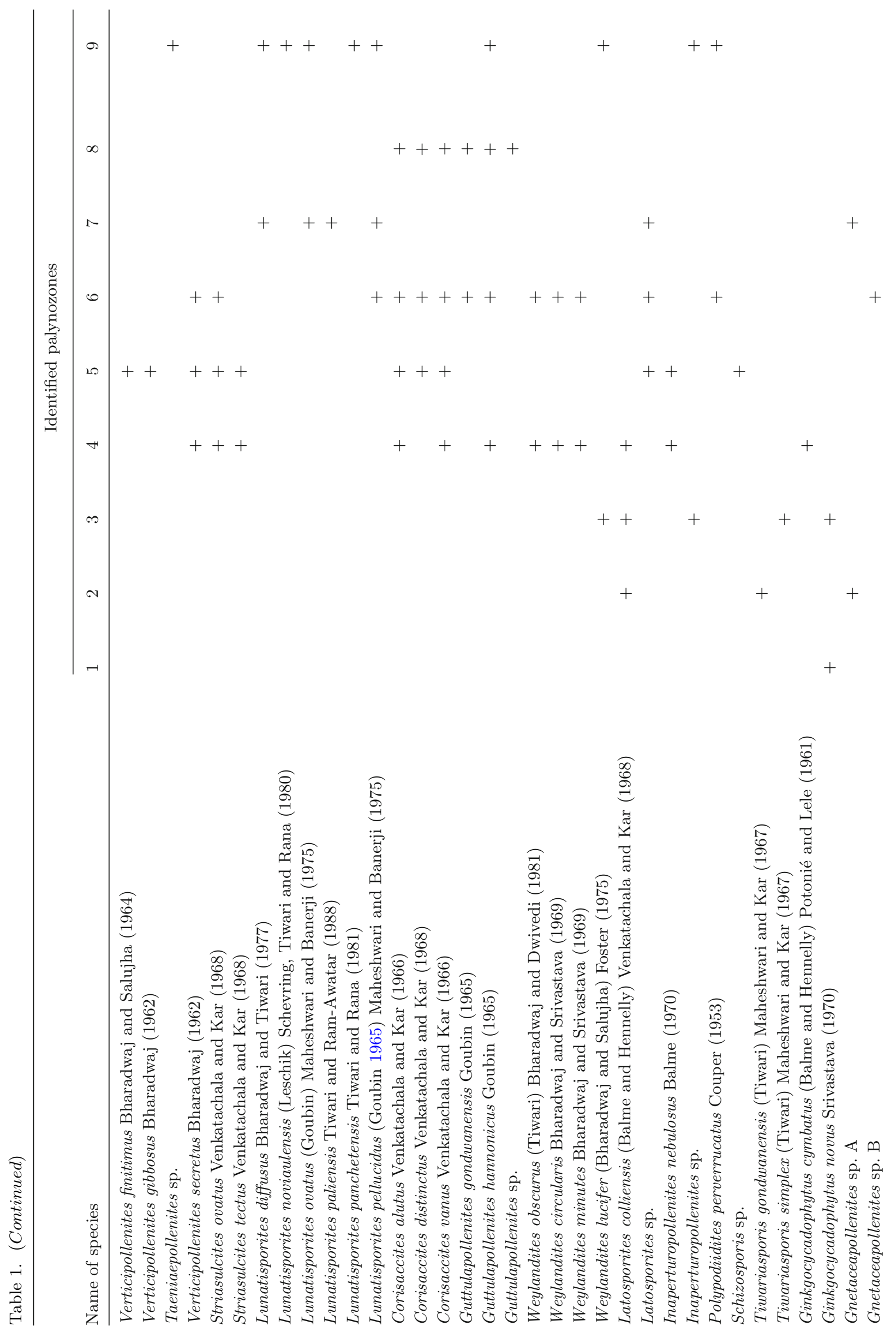




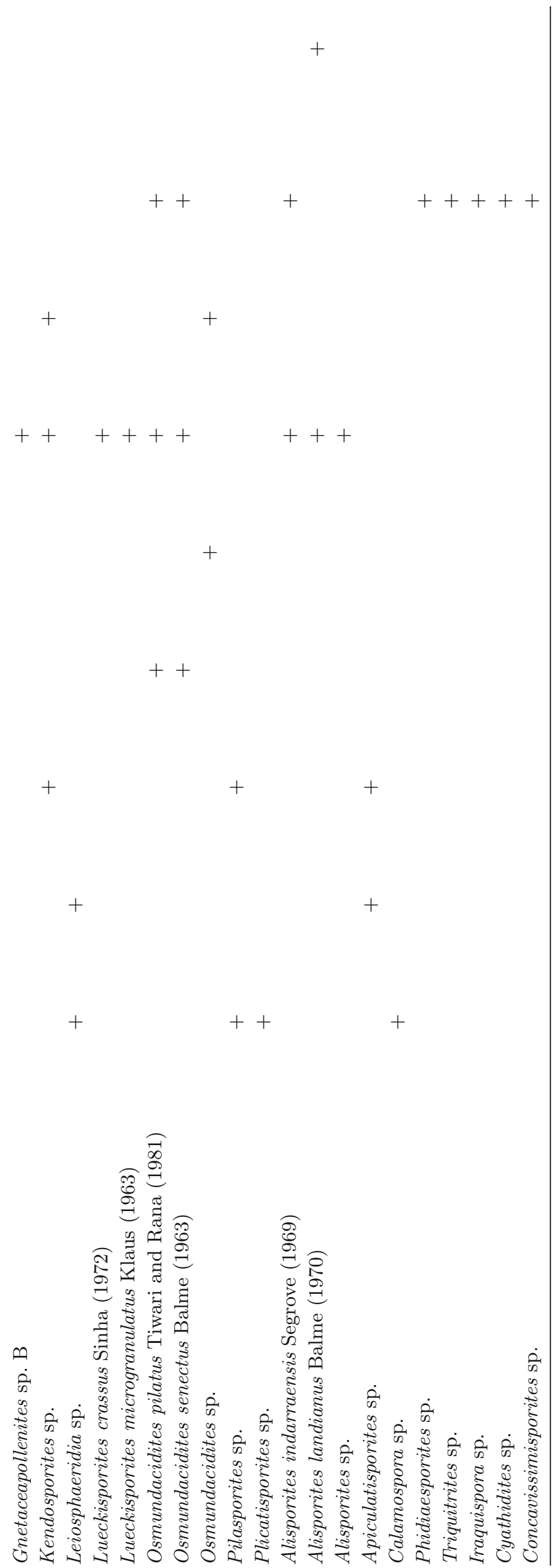

tiwarii, Striatites communis, S. naditolensis, $S$. solitus, Lahirites incertus, L. levicorpus, L. rarus in the palynoassemblages of Palynozone-3 indicate Barakar palynoflora (Early Permian).

Age span: Artinskian age (Jha 2006).

Remarks: This palynozone corresponds with Scheuringipollenites barakarensis assemblage zone of Tiwari and Tripathi (1992) from Damodar Basin.

\subsubsection{Palynozone-4}

Representative Occurrences

Palynozone-4 includes:

- Palynoassemblage-I of borecore GAM-3

- Palynoassemblage-II of borecore GAM-7

- Palynoassemblage-III of borecore GAM-8

Composition: Identified palynoassemblages of Palynozone-4 in having the dominance of striate disaccates, viz., Faunipollenites copiosus, F. goraiensis, F. parvus, Striatopodocarpites brevis, $S$. decorus, S. diffuses, S. globosus, S. labrus, S. multistriatus, S. rotundus, S. subcircularis, S. tiwarii and sub-dominance of non-striate disaccates, viz., Scheuringipollenites, Ibisporites and Vesicaspora along with some stratigraphically significant taxa, viz., Weylandites, Guttulapollenites, Corisaccites, Aurangapollenites and Osmundacidites distinguish this palynozone from others. The first appearance of Densipollenites brevis, D. indicus, D. invisus, D. magnicorpus, Striasulcites ovatus, S. tectus, Corisaccites alutus, C. vanus, Guttulapollenites hannonicus, Weylandites obscures, W. circularis, $W$. minutes makes this palynozone distinct from others. On the basis of palynofloral distribution, Late Permian (Raniganj) age has been assigned to this palynoflora.

Age span: Guadalupian age (Jha 2006).

Remarks: This palynozone compares well with zone Gondisporites raniganjensis (RIIA) of Tiwari and Tripathi (1992).

\subsubsection{Palynozone-5}

Representative Occurrences

Palynozone-5 includes:

- Palynoassemblage-II of borecore GAM-3

- Palynoassemblage-II of borecore GAM-10 
Composition: Dominance of striate disaccate Striasulcites ovatus, S. tectus along with other striates, viz., Faunipollenites bharadwajii, F. copiosus, F. gopadensis, F. goraiensis, F. parvus, Striatopodocarpites brevis, $S$. decorus, $S$. diffuses, $S$. globosus, S. rotundus, S. tiwarii, Lahirites levicorpus, L. rhombicus, L. singularis, Striatites communis, S. obliquus, S. parvus, S. solitus, S. tentulus, Verticipollenites crassus, $V$. debilis, $V$. finitimus, $V$. gibbosus, $V$. secretus and a few non-striate disaccates (Scheuringipollenites, Vesicaspora, Schizopollis) along with stratigraphically significant taxa Circumstriatites obscures, C. ovatus, Corisaccites alutus, $C$. distinctus, C. vanus, Osmundacidites sp. signifies the resemblance of this palynoflora with Raniganj (Late Permian).

Age span: Guadalupian age (Jha 2006).

Remarks: This palynozone has not been identified in Damodar Basin, (Tiwari and Tripathi 1992).

\subsubsection{Palynozone-6}

Representative Occurrences

Palynozone-6 includes:

- Palynoassemblage-III of borecore GAM-3

- Palynoassemblage-III of borecore GAM-7

Composition: Identified palynoassemblages of Palynozone-6 in having the dominance of monosaccate: Densipollenites brevis, D. densus, D. indicus, D. invisus, D. kamthiensis, D. magnicorpus var. annulatus, D. minimus along with some striate disaccates, viz., Faunipollenites bharadwajii, F. copiosus, F. gopadensis, F. goraiensis, F. parvus, F. singrauliensis, F. varius, Striatopodocarpites diffuses, S. globosus, S. rotundus, Crescentipollenites amplus, C. barakarensis, C. fuscus, C. globosus, C. gondwanensis, Lahirites incertus, L. levicorpus, L. rarus, L. rhombicus, Striatites communis, Verticipollenites debilis, $V$. secretus and stratigraphically significant taxa, viz., Gondisporites raniganjensis, Striomonosaccites ovatus, Lunatisporites pellucidus, Corisaccites, Guttulapollenites, Lundbladispora raniganjensis, L. willmotti, Klausipollenites schaubergeri, $K$. vestitus, Corisaccites alutus, $C$. distinctus, C. vanus, Guttulapollenites gondwanensis, G. hannonicus, Weylandites obscurus, W. circularis, W. minutes, Striasulcites ovatus, Polypodiidites perverrucatus, Osmundacidites pilatus, $O$. senectus distinguish this palynozone from others. Palynofloral distribution of these palynoassemblages assigned the Late Permian (Raniganj) age to this palynozone.

Age span: Lopingian age (Jha 2006).

Remarks: This palynozone corresponds well with Densipollenites magnicorpus assemblage zone (RIA) of Damodar Basin (Tripathi and Tiwari 1982).

\subsubsection{Palynozone- 7}

Representative Occurrences

Palynozone-7 includes:

- Palynoassemblage-IV of borecore GAM-7.

Composition: Palynozone- 7 is notable from other palynozones by the good percentage of striate disaccate Crescentipollenites multistriatus, $C$. amplus, C. barakarensis, C. brevis, C. circumstriatites, $C$. densus, $C$. fuscus, $C$. globosus, $C$. gondwanensis, C. implicates, C. talchirensis along with Striatopodocarpites diffuses, $S$. rotundus, Faunipollenites copiosus, F. gopadensis, Lahirites karanpuraensis, L. singularis and stratigraphically significant: Falcisporites nuthaliensis, $F$. stabilis, Lunatisporites diffusus, L. ovatus, $L$. paliensis, L. pellucidus. Palynofloral distribution assigned the Late Permian (Raniganj) age to this palynozone.

Age span: Lopingian age (Jha 2006).

Remarks: This palynozone corresponds Densipollenites magnicorpus assemblage zone (RIB) of Damodar Basin (Tiwari and Tripathi 1992).

\subsubsection{Palynozone-8}

Representative Occurrences

Palynozone-8 includes:

- Palynoassemblage-V of borecore GAM-7 (178.4 m).

Composition: Palynozone- 8 is discriminated from other palynozones by the good percentage of taeniate pollen Guttulapollenites gondwanensis, $G$. hannonicus along with striate disaccates Faunipollenites goraiensis, F. parvus, Striatopodocarpites diffuses, S. globosus, $S$. rotundus, and stratigraphically significant taxa Falcisporites nuthaliensis, Corisaccites alutus, C. distinctus, C. vanus, Osmundacidites pilatus, $O$. senectus, Phidiaesporites sp., Triquitrites sp., Iraquispora sp., 
Cyathidites sp., Concavissimisporites sp. Palynofloral distribution assigned the Late Permian (Raniganj) age to this palynozone.

Age span: Lopingian age (Jha 2006).

Remarks: This palynozone has never been identified in Damodar Basin (Tiwari and Tripathi 1992).

\subsubsection{Palynozone-9}

Representative Occurrences

Palynozone-9 includes:

- Palynoassemblage-VI of borecore GAM-7.

Composition Identified palynoassemblage of Palynozone-9 in having the dominance of taenaite Lunatisporites diffusus, $L$. noviaulensis, L. panchetensis, L. ovatus, L. pellucidus and sub-dominance of triletes, viz., Verrucosisporites surangei, $V$. triassicus, Horriditriletes sp. along with some stratigraphically significant taxa, viz., Lundbladispora microconata, L. raniganjensis, L. willmotti, Playfordiaspora cancellosa, Klausipollenites schaubergeri, Strotersporites decorus, Taeniaepollenites sp., Polypodiidites perverrucatus, Guttatisporites ambiguous, Ringosporites ringus, Guttulapollenites hannonicus and Weylandites lucifer distinguish this palynozone from others. On the basis of palynofloral distribution, the assigned age of this palynoflora is Early Triassic.

Age span: Induan-Olenekian age (Jha 2006).

Remarks: This palynozone corresponds with Playfordiaspora cancellosa (PIII) assemblage zone of Damodar Basin (Tiwari and Tripathi 1992).

\section{Comparison and discussion}

On the basis of dominance and sub-dominance of different spore pollen taxa of monosaccates, nonstriate disaccates, striate disaccates along with some stratigraphically significant taxa, total of 12 assemblages have been identified in Lower Gondwana of Godavari Graben (Jha 2006). In the present investigation, quantitative assessment of various palynomorphs in the borecores GAM-3, GAM-6, GAM-7, GAM-8 and GAM-10 of Maila- ram area have lead to recognize nine palynozones out of which Palynozone-1, 2 and 3 belong to Early Permian (Talchir, Karharbari, Barakar formations). Palynozones 4-8 belong to Late Permian (Raniganj Formation) and Palynozone-9 belongs to Early Triassic (Panchet Formation).

Palynozone-1 is characterized by the dominance of different species of Parasaccites, Plicatipollenites and Caheniasaccites (table 1) along with the presence of Divarisaccus lelei and Jayantisporites pseudozonatus. This palynozone compares well with the Parasaccites korbaensis Assemblage zone (zone I-B) of Tiwari and Tripathi (1992) in having the dominance of monosaccates and presence of Parasaccites korbaensis, Jayantisporites pseudozonatus, Ginkgocycadophytus novus and the first appearence of Divarisaccus lelei. Besides, it has also been represented from different areas, viz., Yellandu (Srivastava 1987), Ramakrishnapuram (Srivastava and Jha 1992a, 1992b), Sattupalli (Srivastava and Jha 1994) of Godavari Graben, Korba Coalfield (Srivastava 1973a, 1973b; Bharadwaj and Srivastava 1973), Giridih Coalfield (Srivastava 1973a, 1973b), Katol area (Kumar and Jha 2000). With other Gondwana continents, Palynozone-1 can also be tentatively correlated with the Parasaccites zone of the Victoria group of Trans-Antarctic Mountain, Antarctica (Truswell 1980); younger part of stage-2 of Eastern Australia; Pseudoreticulatispora confluens zone of Western Australia (Evans 1969; Kemp et al. 1977; Backhouse 1991, 1993) in having the dominance of radial monosaccates; Dwyka Tillite of Africa (Falcon 1975; Anderson 1977; Utting 1978); Darwin Tillite of Antarctica (Barrett et al. 1975); Lower Itarare Group of Brazil (Bharadwaj et al. 1976), Victoria Group Roaring Formation of Antarctica (Kyle and Schopf 1982), Tobra Formation of South America (Masood et al. 1992), Mackellar Formation of Antarctica (Masood et al. 1994).

Palynozone-2 is distinguished by the prominence of different species of Parasaccites along with non-striate disaccate Scheuringipollenites. Besides these, a very good percentage of triletes, viz., Indotriradites (26\%) and Jayantisporites (2-10\%) has also been marked. Horriditriletes ramosus, $H$. rampurensis, Indotriradites korbaensis, I. sparsus, Lophotriletes rectus, Microbaculispora tentula, Parasaccites diffuses, $P$. distinctus, Caheniasaccites distinctus, C. ovatus, Crucisaccites indicus, Scheuringipollenites tentulus, Ibisporites jhingurdahiensis, Platysaccus papillionis, Primuspollenites levis, Faunipollenites parvus, Striatites obliquus, S. parvus, Striatopodocarpites decorus, S. diffuses, Latosporites colliensis, Tiwariasporis gondwanensis, Gnetaceapollenites sp. and Apiculatisporites sp. have been marked for the first time in this 
palynozone. Palynozone-2 corresponds with the Crucisaccites monoletes Assemblage zone of Tiwari and Tripathi (1992) in having Tiwariasporis gondwanensis, Indotriradites korbaensis, Callumispora barakarensis, Parasaccites obscures and P. korbaensis. Similar palynoflora has also been recorded from Manuguru (Srivastava and Jha 1992a, 1992b), Ramakrishnapuram (Srivastava and Jha 1992a, 1992b), Koyagudem of Godavari Graben (Srivastava and Jha 1996), Raniganj Coalfield (Tiwari 1973), Umrer Quarry, Nagpur (Bharadwaj and Anand-Prakash 1974), Pathakhera Coalfield (Sarate 1986; Srivastava and Sarate 1989); Umrer Coalfield (Jha et al. 2007).

Palynozone-3 is discriminated by the dominance of non-striate disaccates, viz., Scheuringipollenites, Ibisporites and sub-dominance of striate disaccates, viz., Faunipollenites, Striatopodocarpites, while Horriditriletes convasus, Cyclogranisporites sp., Parasaccites perfectus, Potonieisporites congoensis, Densipollenites densus, Scheuringipollenites barakarensis, Platysaccus densicorpus, P. plicatus, Sahnites gondwanensis, Rhizomaspora indica, Faunipollenites gopadensis, F. goraiensis, Lahirites incertus, L. levicorpus, L. rarus, Striatites communis, $S$. naditolensis, $S$. solitus, Striatopodocarpites globosus, S. labrus, S. rotundus, S. tiwarii, Verticipollenites crassus, $V$. debilis, Weylandites lucifer, Inaperturopollenites sp., Tiwariasporis simplex and Kendosporites sp. have been marked for the first time in this palynozone. Palynozone-3 shows its equivalence with Scheuringipollenites barakarensis Assemblage zone (zone III A) of Tiwari and Tripathi (1992) in having the dominance of non-striate disaccates, viz., Scheuringipollenites barakarensis, S. maximus, Primuspollenites levis and other taxa, viz., Weylandites lucifer. This palynozone has also been identified from Ramakrishnapuram (Srivastava and Jha 1992a, 1992b), Manuguru (Srivastava and Jha 1992a, 1992b), Budharam (Srivastava and Jha 1995), Koyagudem of Godavari Graben (Srivastava and Jha 1996); Jharia Coalfield (Tripathi and Tiwari 1982); Korba Coalfield (Srivastava 1984), Wardha Coalfield (Bhattacharyya 1997); Sohagpur Coalfield (Ram-Awatar et al. 2003). This palynoflora is tentatively akin to Vesicaspora zone of Ktewaka Coalfield in Tanzania (Manum and Tien 1973); Scheuringipollenites-dominant palynoassemblage of Namwele-Mkomolo and Moze coalfields in Tanzania (Semkiwa et al. 1998).

Palynozone-4 shows the dominance of striate disaccates (Faunipollenites, Striatopodocarpites, Lahirites) and sub-dominance of non-striate disaccates (Scheuringipollenites, Ibisporites and Vesicaspora) along with some stratigraphically significant taxa, viz., Weylandites, Guttulapollenites,
Corisaccites and Osmundacidites which makes it different from other palynozones. Besides these, Leiotriletes rarus, Microbaculispora gondwanensis, Parasaccites bilateralis, P. ovatus, Densipollenites brevis, D. indicus, D. invisus, D. magnicorpus, Scheuringipollenites minutus, Vitreisporites pallidus, Aurangapollenites gurturiensis, A. minimus, Schizopollis extremus, Crescentipollenites barakarensis, C. fuscus, C. globosus, Distriatites distinctus, D. insolitus, Faunipollenites copiosus, Lahirites karanpuraensis, L. singularis, Striatites tentulus, Striatopodocarpites multistriatus, S. subcircularis, Verticipollenites secretus, Striasulcites ovatus, S. tectus, Corisaccites vanus, Guttulapollenites hannonicus, Weylandites obscures, $W$. circularis, $W$. minutes, Inaperturopollenites nebulosus, Ginkgocycadophytus cymbatus, Osmundacidites pilatus, O. senectus have been appeared for the first time in this palynozone. This assemblage zone compares well with Striatopodocarpites + Faunipollenites Assemblage zone (zone V-A; lies under Gondisporites raniganjensis) of Tiwari and Tripathi (1992). This palynozone has also been identified in different areas of Godavari Graben by Srivastava and Jha (1987) in Chelpur; Bharadwaj et al. (1987) in Mantheni; Srivastava and Jha (1992a, 1992b) in Ramakrishnapuram; Srivastava and Jha (1994) in Sattupalli; Srivastava and Jha (1998) in Bhopalpalli and Jha (2004) in Bottapagudem. In addition, similar palynofloras have also been found in Satpura Basin (Bharadwaj et al. 1978a, 1978b), Damodar Valley (Bharadwaj et al. 1979; Tiwari and Singh 1986), South Rewa Basin (Tiwari and Ram-Awatar 1989), Kamptee Coalfield (Srivastava and Bhattacharyya 1996) and Sohagpur Coalfield (Ram-Awatar et al. 2003).

Palynozone-5 is signified by the dominance of striate disaccate Striasulcites along with other striate disaccates, viz., Faunipollenites, Striatopodocarpites and a few non-striate disaccates (Scheuringipollenites, Vesicaspora, Schizopollis). In this palynozone, Circumstriatites obscures, $C$. ovatus, Distriatites insolitus, Lahirites rhombicus, Striatites obliquus, S. parvus and Schizosporis sp. have been marked for the first time. This assemblage zone has been recorded only in Godavari Graben. In this graben, this palynozone has been recovered from other areas, viz., Mantheni area (Bharadwaj et al. 1987), Ramagundam, Khammampalli and Manuguru areas (Srivastava and Jha 1988).

Palynozone-6 is characterized by the dominance of enveloping monosaccate Densipollenites along with some striate disaccates, viz., Faunipollenites, Striatopodocarpites and some stratigraphically significant taxa, viz., Gondisporites, Lundbladispora, Falcisporites, Klausipollenites, 
Striomonosaccites, Corisaccites, Guttulapollenites and Weylandites. Beside it Gondisporites raniganjensis, Lundbladispora raniganjensis, L. willmotti, Densipollenites kamthiensis, D. magnicorpus var. annulatus, D. minimus, Striomonosaccites ovatus, Ibisporites diplosaccus, Falcisporites stabilis, Klausipollenites schaubergeri, $K$. vestitus, Crescentipollenites amplus, C. gondwanensis, Faunipollenites bharadwajii, F. singrauliensis, Lunatisporites pellucidus, Guttulapollenites gondwanensis, Polypodiidites perverrucatus, Gnetaceapollenites sp., Lueckisporites crassus, L. microgranulatus, Alisporites indarraensis, A. landianus have been marked for the first time in this palynozone. The first appearence of Lundbladispora raniganjensis, L. willmotti, Gondisporites raniganjensis, Striomonosaccites ovatus, Falcisporites stabilis, Klausipollenites schaubergeri, Guttulapollenites gondwanensis and Lunatisporites pellucidus are significant stratigraphically as the presence of these species indicates Late Permian age (upper part of Raniganj Formation). Dominance of striate disaccates (Striatopodocarpites, Faunipollenites) and acme of Densipollenites along with the presence of Densipollenites densus, $D$. indicus, $D$. invisus, D. magnicorpus var. annulatus, Klausipollenites schaubergeri, Crescentipollenites fuscus and $C$. gondwanensis makes Palynozone-6 comparable with Striatopodocarpites-Densipollenites Assemblage Zone (zone V-C) or Densipollenites magnicorpus Assemblage zone of Tiwari and Tripathi (1992). Besides, this assemblage zone has also been recovered from other Indian Lower Gondwana basins, viz., Son Valley (Tiwari and Ram-Awatar 1989), Damodar Basin (Bharadwaj et al. 1979; Tiwari and Singh 1983), Satpura Basin (Bharadwaj et al. 1978a, 1978b), Rajmahal Basin (Tiwari and Tripathi 1984), Mahanadi Basin (Tiwari et al. 1991; Tripathi 1997), Kamptee Coalfield (Srivastava and Bhattacharyya 1996).

Palynozone-7 is remarkable from other palynozones by the higher percentage (20\%) of striate disaccate of Crescentipollenites together with, Striatopodocarpites, Faunipollenites and stratigraphically significant Falcisporites, Lunatisporites, Corisaccites. Species appeared for the first time in this palynozone are Crescentipollenites brevis, C. circumstriatites, $C$. densus, C. implicates, $C$. talchirensis, Falcisporites nuthaliensis, Columinisporites sp., Lunatisporites diffusus, L. ovatus and L. paliensis, Columinisporites sp. Palynozone-7 resembles with Striatopodocarpites-Crescentipollenites Zone (Zone V-D)/Densipollenites magnicorpus assemblage zone of Tiwari and Tripathi (1992) in having Densipollenites magnicorpus var. annulatus, Crescentipollenites fuscus, C. gondwanensis and Lunatisporites diffusus. Similar assemblage zone has also been traced by Tiwari and RamAwatar (1989) in Son Valley; Bharadwaj et al. (1979) and Tiwari and Singh (1983) in Damodar Basin; Tiwari et al. (1991) and Tripathi (1997) in Talcheer Coalfield of Mahanadi Basin; Srivastava and Bhattacharyya (1996) in Kamptee Coalfield; Bharadwaj et al. (1978a, 1978b) in Satpura Basin.

Palynozone- 8 is discriminated from other palynozones by $29 \%$ of taeniate Guttulapollenites along with striate disaccate Striatopodocarpites, Faunipollenites and stratigraphically significant taxa, viz., Falcisporites. In this palynozone Verrucosisporites surangei, Strotersporites indicus, S. crassiletus, Phidiaesporites sp., Triquitrites sp., Iraquispora sp., Cyathidites sp., and Concavissimisporites sp. appeared for the first time. This palynozone has never been recorded in Damodar Basin but has been traced by different researchers in other Indian basins, viz., Godavari Graben (Srivastava and Jha 1989, 1990), Satpura basin (Bharadwaj et al. 1978a, 1978b). Palynozone-8 of Godavari Graben also has resemblance with the Late Permian assemblage recorded from other Gondwana continents, viz., Malagasy (Goubin 1965; De Jekhowsky and Goubin 1964; Wright and Askin 1987) in upper part of Sakamena Group; Salt Range (Balme 1970) in Chhidru Formation; Luwega Basin (Hankel 1987) in Sumbadzi Member; Rukwa Basin (Wescott et al. 1991) from Karoo sediments; north Luangwa Valley (Utting 1979) from Lower and Upper Madumabisa mudstone member; Zambesi Basin (Falcon 1975). Guttulapollenites palaeophytogeographic province has been identified in Glossopteris dominated megafloral province of Gondwana in central part of Gondwanaland during Late Permian time extending from Salt Range in the north to Amery Basin (Antarctica) in south, Satpura-Wardha-Godavari Basins (India) in east to Mid-Zambesi-Luangawa Valley (Africa) in the west (Jha 2006).

The dominance of striate disaccate pollen grains along with rare occurrence of stratigraphically significant taxa, viz., Gondisporites, Striomonosaccites, Falcisporites, Klausipollenites, Lunatisporites, Crescentipollenites, Strotersporites, Corisaccites, Guttulapollenites, Aurangapollenites, Ginkgocycadophytus, Osmundacidites and Weylandites is the characteristic association in Palynozone 58. Associated taxa become more important while identifying different palynozones because striate disaccates show dominant and sub-dominant component of the palynoflora from Lower Barakar (Early Permian) to Raniganj (Late Permian) (Bharadwaj 1975) i.e., they are long ranging taxa. Thus, the presence of these stratigraphically significant taxa makes the distinction among them. 
Palynozone-9 of the present investigation is characterized by the dominance of taeniate pollen of Lunatisporites and sub-dominance of triletes, viz., Verrucosisporites, Horriditriletes along with some stratigraphically significant taxa, viz., Klausipollenites, Lundbladispora, Playfordiaspora. In this palynozone, Verrucosisporites triassicus, Lundbladispora microconata, Playfordiaspora cancellosa, Guttatisporites ambiguous, Ringosporites ringus, Striatopodocarpites sp., Strotersporites decorus, Taeniaepollenites sp., Lunatisporites noviaulensis, L. panchetensis appeared for the first time. Occurrence of Lundbladispora microconata, L. rani ganjensis, L. willmotti, Playfordiaspora cancellosa is the characteristics of this palynozone. This palynozone compares well with LunatisporitesVerrucosisporites Zone (Zone VI-C) (Playfordiaspora cancellosa Assemblage zone) of Tiwari and Tripathi (1992) in having a decline in the prominence of striate disaccates and increase in percentage of taeniate disaccates with apiculate triletes. This palynozone compares well with Palynoassemblage-II of Sattupalli area of Godavari Graben (Jha 2008). Recently, Jha et al. (2011) have reported Early Triassic palynoflora in Manuguru area. The palynofossil content of this Early Triassic palynoflora of Manuguru area is dominated by cingulated cavate spores, viz., Lundbladispora and Densoisporites and taeniate pollen, viz., Lunatisporites, in association with other stratigraphically significant taxa such as Falcisporites, Klausipollenites and Chordasporites. Quantitatively, the Manuguru palynoflora shows resemblance with the present Early Triassic palynoflora of Mailaram area (Palynozone-9). But the palynoflora recorded in Manuguru area is younger than the present Early Triassic palynoflora (Palynozone-9) of Mailaram area in view of higher percentage of Lundbladispora and Densoisporites which supercedes the taeniate phase in Damodar Basin (Tiwari and Singh 1986). Outside India, present assemblage zone (Palynozone-9) is comparable to Lunatisporites pellucidus zone (Falcisporites superzone) of Australia (Helby et al. 1987), Early Scythian Assemblage of Mianwali Formation, Salt Range, Western Pakistan (Balme 1970); Early Triassic plant microfossil assemblage of Lower Mariakani Formation, Kenya (Hankel 1990) in having the dominance of Lunatisporites and presence of Lundbladispore, Densoisporites, Playfordiaspora, Klausipollenites with striate disaccates.

\section{Conclusions}

The main concluding remarks derived from the palynological studies on Permian-Triassic sediments of Mailaram area, Godavari Graben reported in this paper are:

- Nine distinct palynozones belonging to Talchir, Karharbari, Barakar, Raniganj and Panchet formations have been identified in Mailaram area.

- Existence of almost complete succession from Early Permian (Talchir)-Panchet (Early Triassic) has been established in Mailaram area.

- A transitional palynostratigraphic contact between Raniganj (Late Permian) and Panchet (Early Triassic) formations has been marked in borecore GAM-7. It contains first appearence of Verrucosisporites triassicus, Lundbladispora microconata, Playfordiaspora cancellosa, Guttatisporites ambiguous, Ringosporites ringus, Strotersporites decorus, Taeniaepollenites sp., Lunatisporites noviaulensis and $L$. panchetensis.

- Karharbari palynoflora has been identified in lithologically designated Barakar Formation and Raniganj palynoflora has been demarcated in lithologically designated Barren Measures Formation.

Table 2. Complete biostratigraphic succession in Mailaram area.

\begin{tabular}{|c|c|c|c|c|}
\hline $\begin{array}{c}\text { Identified } \\
\text { palynozones }\end{array}$ & Borecores & Formation & Period & Stage \\
\hline Palynozone-9 & GAM-7 & Panchet & Triassic & Induan-Olenekian \\
\hline Palynozone-8 & GAM-7 & & & Lopingian \\
\hline Palynozone-7 & GAM-7 & Raniganj & Late Permian & \\
\hline Palynozone- 6 & GAM-3, GAM -7 & & & \\
\hline Palynozone-5 & GAM-3, GAM-10 & Baniogani & L ate Permian & Guadalupian \\
\hline Palynozone- 4 & GAM-3, GAM-7, GAM-8 & naninganj & Lale Perman & \\
\hline Palynozone-3 & GAM-6, GAM-8 & Barakar & Early Permian & Artinskian \\
\hline Palynozone-2 & GAM-7, GAM-8 & Karharbari & & \\
\hline Palynozone-1 & GAM-6, GAM-10 & Talchir & & Asselian-Sakmarian \\
\hline
\end{tabular}


- Barren Measures palynoflora has not been identified in any of the borecore which indicates absence of Barren Measures sediment in Mailaram area. This break up in sediments may be due to either nondeposition or simultaneous erosion of sediments.

- Permian Triassic transition has been observed in borecore GAM-7. Permian/Triassic transition in Mailaram area is similar to Salt Range.

- Presence of Early Triassic palynoflora in borecore GAM-7 indicates occurrence of Panchet sediments in Mailaram area of Godavari Graben.

- Palynologically, Early Permian age has been assigned to Palynozone-1; Palynozone-2, Palynozone-3 and Palynozone-4; Late Permian age has been assigned to Palynozones 4-8; and Early Triassic age has been assigned to Palynozone-9 (table 2).

- The present spore-pollen study has demonstrated the existence of two coal-bearing horizons (Lower coal-bearing horizon: Karharbari and Barakar formations, Upper coal-bering horizon: Raniganj Formation) in Mailaram area of Godavari Graben.

\section{Acknowledgements}

The authors wish to express their sincere thanks to $\mathrm{Dr}$ N C Mehrotra, Director, Birbal Sahni Institute of Palaeobotany for granting permission for the publication of this research. Thanks are extended to the authorities of Geological Survey of India (GSI) for the permission to collect the samples.

\section{References}

Anderson J M 1977 The biostratigraphy of the Permian and Triassic - part-3. A review of Gondwana Permian palynology with particular reference to the northern Karoo Basin, South Africa; Mem. Bot. Surv. South Africa 41 $1-67$.

Backhouse J 1991 Permian palynostratigraphy of the Collie Basin, Western Australia; Rev. Palaeobot. Palynol. 67 237-314.

Backhouse J 1993 Palynology and correlation of Permian sediments in the Perth, Collie, and Officer basins, Western Australia; Geol. Surv. Western Australia Rep. 34 111-128.

Balme B E 1970 Palynology of Permian and Triassic strata in the Salt Range and Surghar Range, Western Pakistan; In: Stratigraphic boundary problems: Permian and Triassic of West Pakistan 4, (eds) Kummel B and Teichert C, Geology Department, University of Kansas, pp. 305-455.

Barrett P J, Kyle and Rosemary A 1975 The Early Permian glacial beds of South Victoria Land and Darwin Mountains, Antarctica; In: Gondwana Geology (ed.) K S W Campbell, Australian National University Press, Canberra, Australia, pp. 333-346.

Bharadwaj D C 1975 Palynology in biostratigraphy and palaeoecology of Indian Lower Gondwana Formations; Palaeobotanist 22 150-157.

Bharadwaj D C and Anand-Prakash 1974 Palynostratigraphy of Lower Gondwana sediments from Umrer Quarry Nagpur, Maharashtra, India; Geophytology 4 130-134.

Bharadwaj D C and Srivastava Suresh C 1973 Sub-surface palynological succession in Korba Coalfield, M.P., India; Palaeobotanist 20 137-151.

Bharadwaj D C, Kar R K and Navale G K B 1976 Palynostratigraphy of Lower Gondwana deposits in Parana and Maranhao basins; Brazil; Biol. Mem. 1 56-103.

Bharadwaj D C, Tiwari R S and Anand-Prakash 1978a A Talchir mioflora from northern Satpura Basin, India; Palaeobotanist 25 62-69.

Bharadwaj D C, Tiwari R S and Anand-Prakash 1978b Palynology of Bijori Formation (Upper Permian) in Satpura Gondwana Basin, India; Palaeobotanist 25 70-78.

Bharadwaj D C, Tiwari R S and Anand-Prakash 1979 Permo-Triassic palynostratigraphy and lithostratigraphical characteristic in Damodar Basin, India; Biol. Mem. 4 49-82.

Bharadwaj D C, Srivastava S C, Ramanamurty B V, Jha Neerja 1987 Palynology of Kamthi Formation from Ramagundam-Mantheni area, Godavari Graben, India; Palaeobotanist 35 318-330.

Bhattacharyya A P 1997 Palynological recognition of the Karharbari-Barakar formations in the sub-surface sediments of Wardha Coalfield, Maharashtra, India; Palaeobotanist 46 217-219.

De Jekhowsky B and Goubin N 1964 Subsurface palynology in Madagascar: A stratigraphic sketch of the Permian, Triassic and Jurassic of the Morondava Basin; In: Palynology in oil Exploration (ed.) Cross A T, Soc. Econ. Paleontol. Mineral. Spec. Publ. 11 116-130.

Evans P R 1969 Upper Carboniferous and Permian palynological stage and their distribution in eastern Australia; In: Gondwana Stratigraphy (ed.) Amos A J, UNESCO, Paris, pp. 41-54.

Falcon R M S 1975 Palynostratigraphy of the Karroo sequence in the central Sebungwe District, Mid Zambesi Basin, Rhodesia; Palaeonto. Africa 18 1-29.

Goubin N 1965 Description et repartition des principaux pollenites permiens, triasiques et jurassique des sondages du sassin de Morondava (Madagascar); Rev. Inst. Fr. Pet. 20 1415-1461.

Hankel O 1987 Lithostratigraphic subdivision of the Karoo rocks of the Luwegu Basin (Tanzania) and their biostratigraphic classification based on microfloras, macrofloras, fossil woods and vertebrates; Geol. Rundsch. 76 539-565.

Hankel O 1990 Early Triassic plant microfossils from the Kavee Quarry section of the Lower Mariakani Formation, Kenya; Rev. Palaeobot. Palynol. 68 127-145.

Helby R J, Morgan R and Par-Tridge A D 1987 A palynological zonation of the Australian Mesozoic; Assoc. Aust. Palaeontol. Memoir 4 1-94.

Jha Neerja 2004 Palynological dating of coal-bearing sediments from the Bottapagudem area, Chintalapudi subbasin, Andhra Pradesh; Palaeobotanist 53 61-67.

Jha Neerja 2006 Permian palynology from India and Africa: A phytogeographical paradigm; J. Palaeontol. Soc. India $5143-55$.

Jha Neerja 2008 Permian-Triassic palynofloral transition in the Sattupalli area, Chintalapudi sub-basin, Godavari 
Graben, Andhra Pradesh, India; J. Palaeontol. Soc. India 52 159-168.

Jha Neerja, Tewari Rajni and Rajanikanth A 2007 Palynology of Permian Gondwana sequence of Umrer Coalfield Maharashtra; J. Geol. Soc. India 69 851-857.

Jha Neerja, Pauline Sabina K and Mehrotra N C 2011 Early Triassic palynomorphs from Manuguru area of Godavari Graben, Andhra Pradesh; J. Geol. Soc. India 78 167-174.

Kemp E M, Blame B E, Helby R A, Playford G and Price P L 1977 Carboniferous and Permian Palynostratigraphy in Australia and Antarctica: A review; BMR J. Aus. Geol. Geophys. 2 177-208.

Kumar P and Jha Neerja 2000 Subsurface palynological succession from Katol area, Nagpur, Maharashtra; Geophytology 29 65-68.

Kyle R A and Schopf J M 1982 Permian and Triassic palynostratigraphy of the Victoria Group, Trans Antarctic Mountains; In: Antarctic Geoscience (ed.) Craddock (Madison: University Winconsin Press), pp. 649-659.

Manum S B and Tien N D 1973 Palynostratigraphy of the Letewaka Coalfield (Lower Permian); Rev. Palaeobot. Palynol. 16 213-227.

Masood K R, Querashi K A and Iqbal M J 1992 Palynostratigraphy of the Gondwana glacial deposits of the Western Salt Range, Pakistan; 1st South Asia Geological Congress Islamabad, 27 (Abstract).

Masood K R, Taylor T N, Horner T and Taylor E L 1994 Palynology of the Mackellar Formation (Beacon Supergroup) of East Antarctica; Rev. Palaeobot. Palynol. 83 329-337.

Ram-Awatar, Mukhopadhyay A and Adhikari S 2003 Palynostratigraphy of subsurface Lower Gondwana, Pali sediments, Sohagpur Coalfield, Madhya Pradesh, India; Palaeobotanist 53 51-59.

Sarate O S 1986 Palynological correlation of the coal seams of Pathakhera Coalfield, Madhya Pradesh, India; Geophytology 16 239-248.

Semkiwa P, Kalkeuth W, Utting J, Mayagilo F, Mpanju F and Hagemann 1998 The geology, petrology, palynology and geochemistry of Permian coal basin in Tanzania. Namwele Mkomolo, Muze Galula Coalfields; Int. J. Coal Geol. 36 63-110.

Srivastava Suresh C 1973a Palynostratigraphy of the Giridih Coalfield; Geophytology 3 84-94.

Srivastava Suresh C 1973b Talchir mioflora from Korba Coalfield, Madhya Pradesh; Geophytology $\mathbf{3}$ 102-105.

Srivastava Suresh C 1984 Palynological correlation of coal seams in Kusmunda block, Korba Coalfield, Madhya Pradesh, India; Palaeobotanist 32 230-235.

Srivastava Suresh C 1987 Palynological correlation of coal seams in Godavari Graben, Andhra Pradesh, India; Palaeobotanist 35 281-296.

Srivastava S C and Bhattacharyya A P 1996 PermianTriassic palynofloral succession in subsurface from Bazargaon, Nagpur District, Maharashtra; Palaeobotanist 43 10-15.

Srivastava S C and Jha Neerja 1988 A Lower Triassic palynoassemblage from Budharam area, Godavari Graben, Andhra Pradesh; Geophytology 18 124-125.

Srivastava S C and Jha Neerja 1989 Palynology of Lower Gondwana sediments in the Godavari Graben, Andhra Pradesh, India; Palaeobotanist 37 199-209.

Srivastava Suresh C and Jha Neerja 1987 Palynology of Kamthi Formation from Chelpur area, Godavari Graben, Andhra Pradesh, India; Palaeobotanist 35 $342-346$.
Srivastava Suresh C and Jha Neerja 1990 Permian-Triassic palynofloral transition in Godavari Graben, Andhra Pradesh; Palaeobotanist 38 92-97.

Srivastava Suresh C and Jha Neerja 1992a Palynostartigraphy of Permian sediments in Manuguru area, Godavari Graben, Andhra Pradesh; In: Proceedings of Birbal Sahni Birth Centenary Palaeobotanical Conference (eds) Vankatachala V S, Jain K P and Awasti N, Geophytology 22 103-110.

Srivastava Suresh C and Jha Neerja 1992b Permian palynostratigraphy in Ramakrishnapuram area, Godavari Graben, Andhra Pradesh, India; Geophytology 20 83-95.

Srivastava Suresh C and Jha Neerja 1994 Palynological dating of Lower Gondwana sediments in Sattupalli area, Chintalapudi sub-basin, Andhra Pradesh, India; Palaeobotanist 42 169-173.

Srivastava Suresh C and Jha Neerja 1995 Palynostratigraphy and correlations of Permian-Triassic sediments in Budharam Area, Godavari Graben, India; J. Geol. Soc. India 46 647-653.

Srivastava Suresh C and Jha Neerja 1996 Palynology of sub-surface Permian sediments in Koyagudem area, Godavari Graben, Andhra Pradesh; Geophytology 25 131-136.

Srivastava Suresh C and Jha Neerja 1998 Palynology of Lower Gondwana sediments in the Bhopalpalli area, Godavari Graben; J. Palaeontol. Soc. India 43 41-48.

Srivastava Suresh C and Sarate O S 1989 Palynostratigraphy of Lower Gondwana sediments from Shobhapur Block, Patherkhera Coalfield, Madhya Pradesh; Palaeobotanist 37 125-133.

Tiwari R S 1973 Palynological succession in the Barakar type area; Geophytology 3 166-183.

Tiwari R S and Ram-Awatar 1989 Sporae dispersae and correlation of Gondwana sediments in Johilla Coalfield, Son Valley Graben, Madhya Pradesh; Palaeobotanist 37 94-114.

Tiwari R S and Singh Vijya 1983 Miofloral transition at Raniganj-Panchet boundary in east Raniganj coalfield and its implication on Permo-Triassic time boundary; Geophytology 13 227-234.

Tiwari R S and Singh Vijya 1986 Palynological evidences for Permo-Triassic boundary in Raniganj Coalfield, Damodar Basin, India; Bull. Geol. Min. Metall. Soc. India 54 256-264.

Tiwari R S and Tripathi A 1984 A report of Raniganj mioflora from sediments of Dubrajpur Formation in Brahmani Coalfield, Rajmahal Basin; Geophytology 14 244-245.

Tiwari R S and Tripathi A 1992 Marker assemblage zones of spore and pollen species through Gondwana Palaeozoic-Mesozoic sequence in India; Palaeobotanist 40 194-236.

Tiwari R S, Tripathi A and Jana B N 1991 Palynological evidence for Upper Permian Raniganj Coals in western part of Talcher Coalfield, Orissa, India; Curr. Sci. 61 407-420.

Tripathi A 1997 Palynostratigraphy and palynofacies analysis of subsurface Permian sediments in Talcher Coalfield, Orissa; Palaeobotanist 46 79-88.

Tripathi A and Tiwari R S 1982 Barakar mioflora from Jharia Coalfield; Geophytology 12 195-200.

Truswell E M 1980 Permo-Carboniferous palynology of Gondwanaland: Progress and problem in the decade to 1980; J. Aust. Geol. Geophys. 5 95-111.

Utting J 1978 Lower Karoo pollen and spore assemblages from the coal measures and underlying sediments of 
the Siankondobo Coalfield, Mid-Zambesi Valley, Zambia; Palynology 2 53-68.

Utting J 1979 Pollen and spore assemblages from the Upper Permian of the North Luangava Valley, Zambia; In: Proceedings of the 4th International Palynology Conference, Moscow, 2 165-174.

Wescott W A, Krebs W N, Engelhardt D W and Cunningham S W 1991 New biostratigraphic age dates from the Lake Rukwa Rift Basin in western Tanzania; Am. Assoc. Petrol. Geol. Bull. 75 1255-1263.

Wright R P and Askin R A 1987 The PermianTriassic boundary in the southern Morondava basin as defined by plant microfossils; In: Gondwana Six Stratigraphy, Sedimentology and Paleontology (ed.) McKenzie G D, Geophys. Monogr., Washington, 41 $157-166$. 